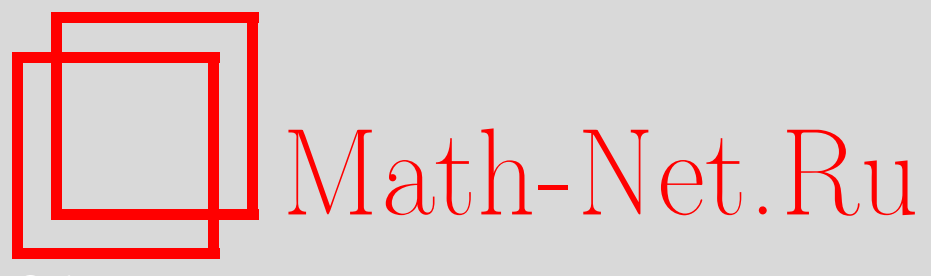

М. А. Васильев, В. Е. Диденко, А. С. Матвеев, Черная дыра БТЗ как решение калибровочной теории полей высших спинов в трехмерном пространстве-времени, TMФ, 2007, том 153, номер 2, 158-185

DOI: https://doi.org/10.4213/tmf6134

Использование Общероссийского математического портала Math-Net.Ru подразумевает, что вы прочитали и согласны с пользовательским соглашением http://www . mathnet.ru/rus/agreement

Параметры загрузки:

IP: 3.93 .64 .190

26 апреля 2023 г., 18:32:40

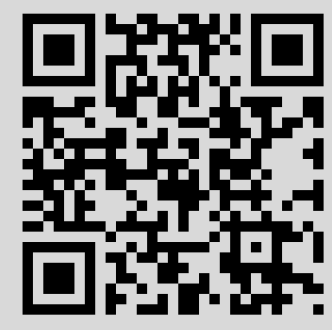




\section{ЧЕРНАЯ ДЫРА БТЗ КАК РЕШЕНИЕ КАЛИБРОВОЧНОЙ ТЕОРИИ ПОЛЕЙ ВЫСШИХ СПИНОВ В ТРЕХМЕРНОМ ПРОСТРАНСТВЕ-ВРЕМЕНИ}

Показано, что черная дыра БТЗ является точным решением калибровочной теории полей высших спинов в трехмерном пространстве-времени. С помощью формализма звездочной алгебры, лежащей в основе теории высших спинов, найдены решения для безмассовых полей в метрике черной дыры. Обнаружено, что при специальных значениях БТЗ-параметров часть симметрий высших спинов остается ненарушенной.

Ключевые слова: черная дыра БТЗ, высшие спины, звездочное произведение.

\section{1. ВВЕДЕНИЕ}

Важное отличие $(2+1)$-мерной теории гравитации [1]-[5] от теорий в более высоком числе измерений заключается в том, что вакуумная теория является топологической, а значит, не описывает никаких локальных степеней свободы. В работах [6], [7] было показано, что $(2+1)$-мерная теория гравитации эквивалентна $S L(2 \mid \mathbb{R}) \times S L(2 \mid \mathbb{R})$-калибровочной теории Черна-Саймонса, калибровочный потенциал которой описывает лоренцеву связность и тетраду. Такая формулировка позволяет рассматривать диффеоморфизмы общей теории относительности как калибровочные преобразования на массовой оболочке, что существенно упрощает квантовый анализ [8].

В трехмерном пространстве тензор Римана полностью выражается через тензор Риччи. Таким образом, из условия $R_{m n}=0$ следует, что $R_{m n p q}=0$, т.е. любое вакуумное решение локально является пространством Минковского. Аналогично любое вакуумное решение уравнений Эйнштейна с отрицательным космологическим членом локально эквивалентно пространству $\mathrm{AdS}_{3}$.

* Физический институт им. П. Н. Лебедева РАН, Москва, Россия. E-mail: vasiliev@lpi.ru, didenko@lpi.ru, matveev@lpi.ru

${ }^{\dagger}$ Museo Storico della Fisica e Centro Studi e Ricerche "Enrico Fermi", Rome, Italy 
Решение типа черной дыры в пространстве $\mathrm{AdS}_{3}$ было найдено в [9]. В работе [10] было показано, что в $(2+1)$-мерном пространстве, в отсутствие отрицательной космологической постоянной, не существует решений с ненулевыми горизонтами событий.

По своим свойствам решение БТЗ во многом аналогично решению Керра в четырех измерениях, что позволяет изучать физику черных дыр на более простом примере. Однако важное отличие заключается в том, что у черной дыры БТЗ отсутствует сингулярность в кривизне [11]. Типичное поведение геодезических диктуется топологической особенностью решения, которое локально изоморфно $\mathrm{AdS}_{3}$. Как было показано в [11], решение БТ3 можно получить факторизацией пространства $\mathrm{AdS}_{3}$ по дискретной подгруппе симметрий.

Поскольку решение БТЗ имеет нулевую $о(2,2)$-кривизну, оно также является и точным решением нелинейной калибровочной теории высших спинов [12], [13], которая в отсутствие материальных полей эквивалентна теории Черна-Саймонса для трехмерной алгебры высших спинов, содержащей подалгебру $o(2,2) \sim s p(2) \oplus$ $s p(2)$. В настоящее время помимо самого пространства AdS известно всего несколько точных решений нелинейной теории высших спинов. Одно из таких трехмерных лоренц-инвариантных решений найдено в работе [13], а его обобщение на четырехмерный случай получено в [14]. К сожалению, их физическая интерпретация по-прежнему отсутствует, несмотря на то что они, по-видимому, играют ключевую роль в теории, являясь базовыми решениями для применения техники интегрирующего потока [13]. Недавно в работе Сезгина и Сандела [15] были найдены новые точные решения, которые могут допускать интерпретацию в контексте AdS/CFT-соответствия.

Безусловно, наиболее важным является исследование решений типа черных дыр в калибровочных теориях высших спинов в высших измерениях. Основная мотивация данной работы заключается в том, что несмотря на простоту случая трех измерений исследование решения БТЗ в калибровочной теории высших спинов может быть полезным для изучения менее тривиальных решений типа Керра-Шварцшильда, как минимум, в двух отношениях. Во-первых, мы изучили приложение техники звездочной алгебры для описания физики черных дыр. Это и является целью данной работы. Во-вторых, можно ожидать, что, так же как четырехмерное пространство Минковского является некоторым срезом плоского десятимерного пространства с матричными координатами [16]-[18] $X^{A B}=X^{B A}$ ( $A, B$ - четырехмерные майорановские спинорные индексы), обычное решение Керра для черной дыры в четырех измерениях можно рассматривать как некий срез БТЗ-подобного решения, связанного с групповым многообразием $S p(4)$, представляющим собой аналог AdS-геометрии согласно [19], [18], [20], [21]. Если это действительно так, то изучение решений нулевой кривизны типа решения БТЗ поможет взглянуть на физику обычных черных дыр с более общей точки зрения многомерных обобщенных пространств с матричными координатами. 
Скромной целью данной работы является демонстрация использования методов теории высших спинов для получения уже известных результатов для черных дыр БТЗ. А именно, с использованием осцилляторной реализации алгебры $o(2,2) \sim s p(2) \oplus s p(2)$ движений пространства $\mathrm{AdS}_{3}$ найдена калибровочная функция решения БТЗ в терминах группы $S p(2)$, а затем решены свободные безмассовые полевые уравнения в метрике БТЗ с помощью формализма модуля Фока [22], тем самым показано, как получить в нашем подходе хорошо известные результаты для скалярного и спинорного полей [23]-[26].

Дальнейшее изложение материала построено следующим образом. В разделе 2 дано краткое описание метрики БТЗ, ее симметрий, а также процедуры факторизации. В разделе 3 представлена осцилляторная реализация алгебры AdS. B разделе 4 дано бескоординатное описание черной дыры БТЗ как плоской связности. БТЗ-калибровочная функция найдена в разделе 5. В разделе 6 обсуждаются $S p(4)$-ковариантные динамические уравнения и их формулировка в терминах модуля Фока [20], [22]. Звездочная реализация векторов Киллинга получена в разделе 7. В разделе 8 , используя развернутую формулировку, мы нашли явные решения для динамических полей в метрике БТЗ. В разделе 9 кратко обсуждается экстремальный случай. И, наконец, в разделе 10 изучаются симметрии безмассовых полей в метрике черной дыры БТЗ. Некоторые полезные формулы и промежуточные вычисления приведены в приложениях А, Б.

\section{2. ЧЕРНАЯ ДЫРА БТЗ}

Кратко напомним основные сведения о черной дыре БТЗ (более подробное изложение можно найти в обзоре [11]).

Из действия Эйнштейна-Гильберта с отрицательной космологической постоянной $\Lambda=-\lambda^{2}$

$$
S=\frac{1}{2 \pi} \int \sqrt{-g}\left(R+2 \lambda^{2}\right) d t d^{2} x
$$

следуют уравнения Эйнштейна

$$
R_{m n}-\frac{1}{2} R g_{m n}=\lambda^{2} g_{m n}
$$

В случае трехмерного пространства-времени имеем

$$
R_{m n p q}=-\lambda^{2}\left(g_{m p} g_{n q}-g_{n p} g_{m q}\right) .
$$

Это означает, что, будучи вакуумным решением, черная дыра локально эквивалентна пространству $\mathrm{AdS}_{3}$. В работе [9] было показано, что метрика

$$
d s^{2}=\left(-M+\lambda^{2} r^{2}+\frac{J^{2}}{4 r^{2}}\right) d t^{2}-\left(-M+\lambda^{2} r^{2}+\frac{J^{2}}{4 r^{2}}\right)^{-1} d r^{2}-r^{2}\left(d \phi-\frac{J}{2 r^{2}} d t\right)^{2},
$$

где $\phi \in[0,2 \pi]$, удовлетворяет уравнению (2.2) и описывает вращающуюся черную дыру с безразмерной массой ${ }^{1)} M$ и угловым моментом $J$. Такая метрика имеет

\footnotetext{
1) Единицы измерения выбраны так, что $G=1 / 8$.
} 
внешний и внутренний горизонты

$$
r_{ \pm}^{2}=\frac{M}{2 \lambda^{2}}\left(1 \pm \sqrt{1-\frac{J^{2} \lambda^{2}}{M^{2}}}\right) .
$$

Эргосфера (т.е. поверхность бесконечного красного смещения $g_{00}=0$ ) имеет радиус $r_{\text {erg }}=M^{1 / 2} / \lambda$.

Заметим, что величины $r_{ \pm}$комплексны при $|J|>M / \lambda$. В этом случае горизонты отсутствуют, а метрика имеет голую сингулярность в точке $r=0$. Формально при $J=0$ можно рассматривать отрицательные значения $M$ в метрике (2.4). Но во всех таких случаях, кроме случая $M=-1$, соответствующего пространству $\mathrm{AdS}_{3}$, это приводит к появлению голых конических сингулярностей при $r=0$ [11], что легко увидеть, переопределив радиальную координату $r \rightarrow \sqrt{-M} r$. Случай $M=0$ и $J=0$ соответствует "безмассовой" черной дыре и не воспроизводит пространство AdS (в отличие от четырехмерного случая). Таким образом, потребуем, чтобы

$$
M>0, \quad|J| \leqslant M / \lambda .
$$

Предельный случай $|J|=M / \lambda$ соответствует экстремальной черной дыре с $r_{+}=r_{-}$.

Пространство $\mathrm{AdS}_{3}$ может быть описано как гиперповерхность, вложенная в четырехмерное псевдоевклидово пространство с метрикой $\eta=\operatorname{diag}(++--)$

$$
\begin{gathered}
d s^{2}=d u^{2}+d v^{2}-d x^{2}-d y^{2}, \\
u^{2}+v^{2}-x^{2}-y^{2}=\lambda^{-2} .
\end{gathered}
$$

В общем случае метрику черной дыры БТЗ (2.4) при $r>r_{+}$можно получить, используя следующую параметризацию:

$$
\begin{aligned}
u & =\sqrt{A(r)} \operatorname{ch}(\tilde{\phi}(t, \phi)), & v & =\sqrt{B(r)} \operatorname{sh}(\tilde{t}(t, \phi)), \\
x & =\sqrt{A(r)} \operatorname{sh}(\tilde{\phi}(t, \phi)), & & y=\sqrt{B(r)} \operatorname{ch}(\tilde{t}(t, \phi)),
\end{aligned}
$$

где

$$
\begin{array}{rlrl}
A(r) & =\frac{1}{\lambda^{2}}\left(\frac{r^{2}-r_{-}^{2}}{r_{+}^{2}-r_{-}^{2}}\right), & & B(r)=\frac{1}{\lambda^{2}}\left(\frac{r^{2}-r_{+}^{2}}{r_{+}^{2}-r_{-}^{2}}\right), \\
\tilde{t} & =\lambda^{2} r_{+} t-\lambda r_{-} \phi, & \tilde{\phi}=-\lambda^{2} r_{-} t+\lambda r_{+} \phi .
\end{array}
$$

В этой работе мы будем рассматривать общий случай и использовать формулы вложения (2.9) при $r>r_{+}$(подробности вложения при $r \leqslant r_{+}$, а также случаи экстремальной и вакуумной черных дыр можно найти в [11]).

Свойства черной дыры БТЗ существенным образом определяются ее групповым происхождением. В самом деле, $(u, v, x, y)$ можно собрать в $(2 \times 2)$-матрицу $S_{0} \in S L(2 \mid \mathbb{R}):$

$$
S_{0}=\lambda\left(\begin{array}{cc}
u+x & v-y \\
-v-y & u-x
\end{array}\right), \quad \operatorname{det} S_{0}=1
$$


Как показано в [11], решение БТЗ получается из группового многообразия $S L(2 \mid \mathbb{R})$ в результате факторизации по дискретной подгруппе при помощи следующего отождествления:

$$
S_{0} \sim \rho^{+} S_{0} \rho^{-}, \quad \rho^{ \pm}=\left(\begin{array}{cc}
e^{\pi \lambda\left(r_{+} \pm r_{-}\right)} & 0 \\
0 & e^{-\pi \lambda\left(r_{+} \pm r_{-}\right)}
\end{array}\right),
$$

которое делает переменную $\phi$ в метрике (2.4) периодической.

Изометрии пространства $\mathrm{AdS}_{3}$ представляются элементами группы $S L(2 \mid \mathbb{R})_{\mathrm{L}} \times$ $S L(2 \mid \mathbb{R})_{\mathrm{R}} / \mathbb{Z}_{2} \sim S O(2,2)$ и действуют на группе левыми и правыми умножениями $S_{0} \rightarrow P_{\mathrm{L}} S_{0} P_{\mathrm{R}}$ по модулю отождествления $\left(P_{\mathrm{L}}, P_{\mathrm{R}}\right) \sim\left(-P_{\mathrm{L}},-P_{\mathrm{R}}\right)$. В соответствии с (2.7) пространство $\mathrm{AdS}_{3}$ инвариантно относительно $S O(2,2)$-преобразований, генераторы которых имеют вид

$$
J_{a b}=X_{b} \frac{\partial}{\partial X^{a}}-X_{a} \frac{\partial}{\partial X^{b}}, \quad X^{a}=(u, v, x, y) .
$$

Согласно [11] в общем случае алгебра движений метрики БТЗ (2.4) определяется векторными полями $\partial / \partial t$ и $\partial / \partial \phi$. В случае $r_{+}^{2}-r_{-}^{2}>0$ вектор Киллинга, ответственный за отождествление (2.13), имеет вид

$$
\frac{\partial}{\partial \phi}=-\lambda r_{+} J_{12}+\lambda r_{-} J_{03}
$$

а генератор сдвига по времени

$$
\frac{\partial}{\partial t}=\lambda^{2} r_{-} J_{12}-\lambda^{2} r_{+} J_{03}
$$

Заметим, что, как показано в [11], среди шести векторов Киллинга пространства $\mathrm{AdS}_{3}$ только (2.15) и (2.16) остаются глобально определенными при отождествлении (2.13).

\section{3. ОСЦИЛЛЯТОРНАЯ РЕАЛИЗАЦИЯ АЛГЕБРЫ $o(2,2)$}

Рассмотрим осцилляторную реализацию алгебры $o(2,2)$, которая будет исключительно важна для дальнейшего анализа. Алгебра движений пространства $\mathrm{AdS}_{3}$ изоморфна алгебре $o(2,2) \sim s p(2) \oplus s p(2)$. Она состоит из диагональных элементов алгебры $s p(2)$ - лоренцевых генераторов $L_{\alpha \beta}=L_{\beta \alpha}$ и AdS-трансляций $P_{\alpha \beta}=P_{\beta \alpha}$ $(\alpha, \beta, \ldots=1,2)$. Коммутационные соотношения имеют вид

$$
\begin{aligned}
& {\left[L_{\alpha \beta}, L_{\gamma \delta}\right]=\frac{1}{2}\left(\epsilon_{\beta \gamma} L_{\alpha \delta}+\epsilon_{\beta \delta} L_{\alpha \gamma}+\epsilon_{\alpha \gamma} L_{\beta \delta}+\epsilon_{\alpha \delta} L_{\beta \gamma}\right),} \\
& {\left[P_{\alpha \beta}, P_{\gamma \delta}\right]=2 \lambda^{2}\left(\epsilon_{\beta \gamma} L_{\alpha \delta}+\epsilon_{\beta \delta} L_{\alpha \gamma}+\epsilon_{\alpha \gamma} L_{\beta \delta}+\epsilon_{\alpha \delta} L_{\beta \gamma}\right),} \\
& {\left[L_{\alpha \beta}, P_{\gamma \delta}\right]=\frac{1}{2}\left(\epsilon_{\beta \gamma} P_{\alpha \delta}+\epsilon_{\beta \delta} P_{\alpha \gamma}+\epsilon_{\alpha \gamma} P_{\beta \delta}+\epsilon_{\alpha \delta} P_{\beta \gamma}\right),}
\end{aligned}
$$

где $\epsilon_{\alpha \beta}=\left(\begin{array}{cc}0 & 1 \\ -1 & 0\end{array}\right)$ - антисимметричная $s p(2)$-инвариантная форма ${ }^{2)}$.

\footnotetext{
${ }^{2)}$ Спинорные индексы поднимаются и опускаются согласно правилу $A_{\alpha}=A^{\beta} \epsilon_{\beta \alpha}, A^{\alpha}=\epsilon^{\alpha \beta} A_{\beta}$.
} 
Пусть заданы осцилляторы $\hat{a}_{\alpha}$ и $\hat{b}^{\alpha}$, удовлетворяющие коммутационным соотношениям

$$
\left[\hat{a}_{\alpha}, \hat{b}^{\beta}\right]=\delta_{\alpha}^{\beta}, \quad\left[\hat{a}_{\alpha}, \hat{a}_{\beta}\right]=0, \quad\left[\hat{b}^{\alpha}, \hat{b}^{\beta}\right]=0 .
$$

Генераторы алгебры $s p(2) \oplus s p(2)$ имеют стандартное осцилляторное представление [27]:

$$
\hat{L}_{\alpha}^{\beta}=\frac{1}{2}\left\{\hat{a}_{\alpha}, \hat{b}^{\beta}\right\}-\frac{1}{4}\left\{\hat{a}_{\gamma}, \hat{b}^{\gamma}\right\} \delta_{\alpha}^{\beta}, \quad \widehat{P}_{\alpha \beta}=\hat{a}_{\alpha} \hat{a}_{\beta}+\lambda^{2} \hat{b}_{\alpha} \hat{b}_{\beta} .
$$

Вместо работы с операторами удобнее использовать коммутирующие переменные $a_{\alpha}$ и $b^{\alpha}$, порождающие ассоциативную алгебру полиномов с операцией звездочного произведения

$$
(f \star g)(a, b)=\frac{1}{\pi^{4}} \int f(a+u, b+t) g(a+s, b+v) e^{2\left(s_{\alpha} t^{\alpha}-u_{\alpha} v^{\alpha}\right)} d^{2} u d^{2} t d^{2} s d^{2} v,
$$

или, что эквивалентно,

$$
(f \star g)(a, b)=f(a, b) \exp \left\{\frac{1}{2}\left(\overleftarrow{\frac{\partial}{\partial a_{\alpha}}} \overrightarrow{\frac{\partial}{\partial b^{\alpha}}}-\overleftarrow{\frac{\partial}{\partial b^{\alpha}}} \vec{\partial}\right)\right\} g(a, b) .
$$

Определенное таким образом звездочное произведение (часто называемое произведением Мояла) описывает ассоциативное произведение симметризованных (упорядоченных по Вейлю) полиномов от осцилляторов в терминах символов операторов. Интеграл нормирован так, что 1 является единичным элементом в алгебре. Таким образом,

$$
1 \star 1=\frac{1}{\pi^{4}} \int e^{2\left(s_{\alpha} t^{\alpha}-u_{\alpha} v^{\alpha}\right)} d^{2} u d^{2} t d^{2} s d^{2} v=1 .
$$

Из (3.4) следует, что

$$
\begin{aligned}
& a_{\alpha} \star f(a, b)=a_{\alpha} f(a, b)+\frac{1}{2} \frac{\partial}{\partial b^{\alpha}} f(a, b), \\
& b_{\alpha} \star f(a, b)=b_{\alpha} f(a, b)+\frac{1}{2} \frac{\partial}{\partial a^{\alpha}} f(a, b) .
\end{aligned}
$$

В частности, определяющие соотношения ассоциативной звездочной алгебры имеют вид

$$
\left[a_{\alpha}, b^{\beta}\right]_{\star}=\delta_{\alpha}^{\beta}, \quad\left[a_{\alpha}, a_{\beta}\right]_{\star}=0, \quad\left[b^{\alpha}, b^{\beta}\right]_{\star}=0,
$$

где $[a, b]_{\star}=a \star b-b \star a$. Звездочная реализация генераторов алгебры $o(2,2)$ имеет вид

$$
L_{\alpha \beta}=\frac{1}{2}\left(a_{\alpha} b_{\beta}+a_{\beta} b_{\alpha}\right), \quad P_{\alpha \beta}=a_{\alpha} a_{\beta}+\lambda^{2} b_{\alpha} b_{\beta} .
$$

Далее везде для удобства будем полагать радиус пространства AdS равным единице $(\lambda=1)$. 


\section{4. РЕШЕНИЕ БТЗ КАК ПЛОСКАЯ СВЯЗНОСТЬ}

Поскольку пространство черной дыры БТЗ локально эквивалентно пространству $\mathrm{AdS}_{3}$, его можно описать, используя плоскую связность алгебры $s p(2) \oplus s p(2)$. Действительно, пусть $w_{0}$ является 1-формой, принимающей значения в алгебре $s p(2) \oplus s p(2)$ :

$$
w_{0}(a, b \mid X)=\frac{1}{2} \omega^{\alpha \beta}(X) L_{\alpha \beta}+\frac{1}{4} h^{\alpha \beta}(X) P_{\alpha \beta},
$$

где $L_{\alpha \beta}$ и $P_{\alpha \beta}-\mathrm{AdS}_{3}$-генераторы $(3.6)$, а $\omega_{\alpha \beta}(X)$ и $h_{\alpha \beta}(X)-1$-формы. Тогда условие нулевой кривизны

$$
R=d w_{0}-w_{0} \star \wedge w_{0}=0
$$

эквивалентно следующим уравнениям:

$$
\begin{aligned}
& d \omega_{\alpha \beta}+\frac{1}{2} \omega_{\alpha}^{\gamma} \wedge \omega_{\gamma \beta}+\frac{1}{2} h_{\alpha}^{\gamma} \wedge h_{\beta \gamma}=0, \\
& d h_{\alpha \beta}+\frac{1}{2} \omega_{\alpha}^{\gamma} \wedge h_{\gamma \beta}+\frac{1}{2} \omega_{\beta}^{\gamma} \wedge h_{\alpha \gamma}=0 .
\end{aligned}
$$

После отождествления $\omega_{\alpha \beta}$ с лоренцевой связностью, а $h_{\alpha \beta}-$ с тетрадой, уравнение (4.4) означает условие отсутствия кручения, а (4.3) локально описывает AdS-геометрию.

Уравнение (4.2) инвариантно относительно калибровочных преобразований

$$
\delta w_{0}=d \epsilon-\left[w_{0}, \epsilon\right]_{\star},
$$

где $\epsilon(a, b \mid X)$ - произвольный инфинитезимальный калибровочный параметр. Любое фиксированное вакуумное решение $w_{0}$ уравнения (4.2) нарушает локальную симметрию до подалгебры стабильности с инфинитезимальным параметром $\epsilon_{0}(a, b \mid X)$, удовлетворяющим уравнению

$$
d \epsilon_{0}-\left[w_{0}, \epsilon_{0}\right]_{\star}=0
$$

Это уравнение совместно вследствие (4.2). Общее решение имеет не более шести независимых параметров глобальных симметрий. Количество таких симметрий, сохраняющихся в локальной AdS-геометрии, зависит от глобальных свойств пространства (т.е. от граничных условий). Настоящее пространство $\mathrm{AdS}_{3}$ обладает всеми шестью симметриями - $o(2,2)$-движениями $\mathrm{AdS}_{3}$. В пространстве черной дыры БТЗ остаются только два из шести таких параметров.

В общем случае тетрада и лоренцева связность алгебры $s p(2) \oplus s p(2)$, удовлетворяющие уравнениям (4.3) и (4.4), локально имеют вид

$$
\begin{aligned}
& h_{\alpha \beta}=\left(W_{1}^{-1}\right)_{\alpha}^{\gamma} d\left(W_{1}\right)_{\gamma \beta}-\left(W_{2}\right)_{\alpha}^{\gamma} d\left(W_{2}^{-1}\right)_{\gamma \beta}, \\
& \omega_{\alpha \beta}=\left(W_{1}^{-1}\right)_{\alpha}^{\gamma} d\left(W_{1}\right)_{\gamma \beta}+\left(W_{2}\right)_{\alpha}^{\gamma} d\left(W_{2}^{-1}\right)_{\gamma \beta},
\end{aligned}
$$

где $W_{1,2 \alpha}{ }^{\beta}(X) \in S p(2)$, т.е.

$$
\left(W_{1,2}^{-1}\right)_{\alpha \beta}=-\left(W_{1,2}\right)_{\beta \alpha} .
$$


Из (4.7) следует, что метрику можно записать как

$$
d s^{2}=\frac{1}{2} h_{\alpha \beta} h^{\alpha \beta}=\frac{1}{2} d S_{\alpha \beta} d S^{\alpha \beta},
$$

где

$$
S_{\alpha \beta}=\left(W_{1}\right)_{\alpha}^{\gamma}\left(W_{2}\right)_{\gamma \beta} .
$$

Таким образом, любая локальная $\mathrm{AdS}_{3}$-метрика определяется $S p(2)$-матричным полем $S_{\alpha \beta}(X)$ (заметим, что в общем случае $S_{\alpha \beta} \neq S_{\beta \alpha}$ ). Чтобы получить метрику БТЗ (2.4), можно использовать матрицу $S_{0}(2.12)$ и параметризацию $(2.9)$.

Семейство хорошо определенных при отождествлении $\phi \rightarrow \phi+2 \pi$ тетрад (4.7) и лоренцевых связностей (4.8) может быть найдено при помощи следующего разложения матрицы $S_{0}(2.12)$ :

$$
S_{0 \alpha}^{\beta}=\left(K_{+} U_{r} K_{-}\right)^{\beta}{ }^{\beta}
$$

по $S p(2)$-матрицам $K_{ \pm}$и $U_{r}$ вида

$$
K_{ \pm}=\left(\begin{array}{cc}
e^{(\tilde{\phi} \mp \tilde{t}) / 2} & 0 \\
0 & e^{-(\tilde{\phi} \mp \tilde{t}) / 2}
\end{array}\right), \quad U_{r}=\left(\begin{array}{cc}
\sqrt{A} & -\sqrt{B} \\
-\sqrt{B} & \sqrt{A}
\end{array}\right) .
$$

Заметим, что матрицы $K_{ \pm}$принадлежат абелевой БТЗ-подгруппе группы $S p(2) \times$ $S p(2)$.

Полагая, что $W_{1}=K_{+} U_{1}$ и $W_{2}=U_{2} K_{-}$, где $U_{1} U_{2}=U_{r}$, мы воспроизводим (4.12) в виде (4.11). Соответствующие тетрада и лоренцева связность

$$
\begin{aligned}
& h=U_{1}^{-1} K_{+}^{-1} d K_{+} U_{1}-U_{2} K_{-} d K_{-}^{-1} U_{2}^{-1}+U_{1}^{-1} d U_{1}-U_{2} d U_{2}^{-1}, \\
& \omega=U_{1}^{-1} K_{+}^{-1} d K_{+} U_{1}+U_{2} K_{-} d K_{-}^{-1} U_{2}^{-1}+U_{1}^{-1} d U_{1}+U_{2} d U_{2}^{-1}
\end{aligned}
$$

не зависят от $t$ и $\phi$ при условии, что $U_{1,2}=U_{1,2}(r)$. Поэтому они остаются хорошо определенными при отождествлении $\phi \rightarrow \phi+2 \pi$ в случае метрики БТЗ.

Удобно выбрать следующие матрицы $U_{1,2}$ :

$$
\begin{aligned}
U_{1} & =\left(\frac{A}{B}\right)^{1 / 4}\left(\begin{array}{cc}
0 & -\mu(r) \sqrt{B} \\
\eta(r) \sqrt{A} & \mu(r) \sqrt{A}
\end{array}\right), \\
U_{2} & =\left(\frac{A}{B}\right)^{1 / 4}\left(\begin{array}{cc}
\mu(r) & 0 \\
-\mu^{-1}(r) & \eta(r) \sqrt{A B}
\end{array}\right),
\end{aligned}
$$

где $\mu(r)$ и $\eta(r)$ - некоторые функции, зависящие от радиальной координаты и удовлетворяющие условию

$$
\mu(r) \eta(r)=A^{-1}(r)
$$

Тогда матрицы $W_{1 \alpha^{\beta}}=\left(K_{+} U_{1}\right)_{\alpha}{ }^{\beta}, W_{2 \alpha}{ }^{\beta}=\left(U_{2} K_{-}\right)_{\alpha}{ }^{\beta}$ принимают вид

$$
\begin{aligned}
W_{1 \alpha}{ }^{\beta} & =\sqrt{\frac{u+x}{y-v}}\left(\begin{array}{cc}
0 & -\mu(y-v) \\
\eta(u-x) & \mu(u-x)
\end{array}\right), \\
W_{2 \alpha}{ }^{\beta} & =\sqrt{\frac{u+x}{y-v}}\left(\begin{array}{cc}
\mu & 0 \\
-\mu^{-1} & \eta(u-x)(y-v)
\end{array}\right) .
\end{aligned}
$$


Согласно (4.7) и (4.8) компоненты тетрады и лоренцевой связности равны

$$
\begin{aligned}
& h_{11}=A \mu^{2}\left(-d \tilde{t}+d \tilde{\phi}+\frac{1}{2 A B} d A\right), \\
& h_{12}=h_{21}=d \tilde{t}-\frac{1}{2 B} d A, \\
& h_{22}=-\mu^{-2}\left(d \tilde{t}+d \tilde{\phi}-\frac{1}{2 A B} d A\right), \\
& \omega_{11}=A \mu^{2}\left(-d \tilde{t}+d \tilde{\phi}+\frac{1}{2 A B} d A\right), \\
& \omega_{12}=\omega_{21}=-d \tilde{\phi}-\frac{1}{2 A} d A-\frac{2}{\mu} d \mu, \\
& \omega_{22}=\mu^{-2}\left(d \tilde{t}+d \tilde{\phi}-\frac{1}{2 A B} d A\right),
\end{aligned}
$$

где $A, B$ и $\tilde{\phi}, \tilde{t}$ определены в $(2.10)$ и $(2.11)$, соответственно. Эти выражения хорошо определены на окружности $S^{1}$ с циклической координатой $\phi \sim \phi+2 \pi$.

\section{5. КАЛИБРОВОЧНАЯ ФУНКЦИЯ}

Уравнение (4.2) локально имеет калибровочное решение вида

$$
w_{0}(a, b \mid X)=-g^{-1}(a, b \mid X) \star d g(a, b \mid X),
$$

где $g(a, b \mid X)$ - некоторый обратимый $\left(g^{-1} \star g=g \star g^{-1}=1\right)$ элемент звездочной алгебры. Как только получена калибровочная функция $g(a, b \mid X)$ в развернутой формулировке, мы сразу же имеем полное решение линеаризованной задачи. В частности, параметры глобальной симметрии, удовлетворяющие уравнению (4.6), имеют вид

$$
\epsilon_{0}(a, b \mid X)=g^{-1}(a, b \mid X) \star \xi \star g(a, b \mid X),
$$

где $\xi=\xi(a, b)$ - произвольный не зависящий от $X$ элемент звездочной алгебры. В разделе 6 будет показано, как знание функции $g(a, b \mid X)$ позволяет получить общее решение свободных полевых уравнений.

Используя результаты [20], мы получаем следующую калибровочную функцию $g\left(a, b \mid W_{1}, W_{2}\right)$, воспроизводящую (4.7) и (4.8) через (5.1):

$$
\begin{aligned}
g\left(a, b \mid W_{1}, W_{2}\right)= & \frac{4}{\sqrt{\operatorname{det}\left\|\left(W_{1}+1\right)\left(W_{2}+1\right)\right\|}} \times \\
g^{-1}\left(a, b \mid W_{1}, W_{2}\right)= & \frac{\times \exp \left(-\frac{1}{2} \Pi^{\alpha \beta}\left(W_{1}\right) T_{\alpha \beta}^{+}-\frac{1}{2} \Pi^{\alpha \beta}\left(W_{2}\right) T_{\alpha \beta}^{-}\right),}{\sqrt{\operatorname{det}\left\|\left(W_{1}+1\right)\left(W_{2}+1\right)\right\|}} \times \\
& \times \exp \left(\frac{1}{2} \Pi^{\alpha \beta}\left(W_{1}\right) T_{\alpha \beta}^{+}+\frac{1}{2} \Pi^{\alpha \beta}\left(W_{2}\right) T_{\alpha \beta}^{-}\right),
\end{aligned}
$$


где $\mathrm{e}^{3)}$

$$
\Pi_{\alpha \beta}(W)=\Pi_{\beta \alpha}(W)=\left(\frac{W-1}{W+1}\right)_{\alpha \beta}
$$

и

$$
T_{\alpha \beta}^{ \pm}=a_{\alpha} a_{\beta}+b_{\alpha} b_{\beta} \pm\left(a_{\alpha} b_{\beta}+b_{\alpha} a_{\beta}\right) .
$$

Здесь $T_{\alpha \beta}^{ \pm}$- генераторы $s p(2)$-подалгебр алгебры $s p(2) \oplus s p(2)$, задаваемые двумя взаимно коммутирующими наборами осцилляторов $\alpha_{\alpha}^{ \pm}=a_{\alpha} \pm b_{\alpha}$ и подчиненные коммутационными соотношениям $\left[\alpha_{\alpha}^{ \pm}, \alpha_{\beta}^{ \pm}\right]_{\star}= \pm 2 \epsilon_{\alpha \beta}$. На практике часто бывает удобнее использовать звездочное произведение, определенное через взаимно коммутирующие осцилляторы $\alpha_{\beta}^{ \pm}$следующим образом ${ }^{4)}$ :

$$
(f * g)\left(\alpha^{ \pm}\right)=\frac{1}{(2 \pi)^{2}} \int f\left(\alpha^{ \pm}+u\right) g\left(\alpha^{ \pm}+v\right) e^{\mp u_{\alpha} v^{\alpha}} d^{2} u d^{2} v
$$

Принимая во внимание, что $T_{\alpha \beta}^{ \pm}=\alpha_{\alpha}^{ \pm} \alpha_{\beta}^{ \pm}$, получаем следующую полезную формулу для произведения калибровочных функций (5.3), которая следует из (5.6) (подробнее см. [20]):

$$
g\left(a, b \mid K_{1}, K_{2}\right) \star g\left(a, b \mid U_{1}, U_{2}\right)=g\left(a, b \mid K_{1} U_{1}, U_{2} K_{2}\right)
$$

при условии, что матрицы $K_{1,2}+1$ и $U_{1,2}+1$ невырожденны. Используя равенство

$$
\Pi_{\alpha \beta}(W)=-\Pi_{\alpha \beta}\left(W^{-1}\right),
$$

находим, что преобразование калибровочной функции (5.3) вида

$$
\begin{gathered}
g\left(a, b \mid W_{1}, W_{2}\right) \rightarrow g\left(a, b \mid W_{1}, W_{2}\right) \star \Lambda^{-1}(a, b \mid V), \\
\Lambda(a, b \mid V)=\frac{4}{\operatorname{det}\|V+1\|} \exp \left(\frac{1}{2} \Pi^{\alpha \beta}(V)\left(T_{\alpha \beta}^{+}-T_{\alpha \beta}^{-}\right)\right), \\
\Lambda^{-1}(a, b \mid V)=\frac{4}{\operatorname{det}\|V+1\|} \exp \left(-\frac{1}{2} \Pi^{\alpha \beta}(V)\left(T_{\alpha \beta}^{+}-T_{\alpha \beta}^{-}\right)\right),
\end{gathered}
$$

где $V_{\alpha \beta}(X) \in S p(2)$, описывает локальное преобразование Лоренца для тетрады (4.7)

$$
h_{\alpha \beta} \rightarrow V_{\alpha}^{\gamma} V_{\beta}^{\delta} h_{\gamma \delta},
$$

оставляя инвариантной метрику (4.10).

Также из (5.7) следует, что тетрада (4.14) и лоренцева связность (4.15) получаются из калибровочной функции вида

$$
g(a, b \mid t, \phi, r)=\Phi(a, b \mid t, \phi) \star U(a, b \mid r),
$$

3) Матричное отношение $B / A$ надо понимать как $A^{-1} B$. Заметим, что соотношение (5.4) аналогично так называемому преобразованию Кэлли.

4) Заметим, что линейные преобразования образующих элементов алгебры Вейля задают автоморфизмы звездочной алгебры, что является следствием определения алгебры Вейля как результата полной симметризации образующих осцилляторов, нечувствительного к определенному выбору базиса в алгебре осцилляторов. 
где

$$
\begin{aligned}
\Phi(a, b \mid t, \phi) & =\frac{4}{\sqrt{\operatorname{det}\left\|\left(K_{+}+1\right)\left(K_{-}+1\right)\right\|}} \exp \left(-\frac{1}{2} \Pi^{\alpha \beta}\left(K_{+}\right) T_{\alpha \beta}^{+}-\frac{1}{2} \Pi^{\alpha \beta}\left(K_{-}\right) T_{\alpha \beta}^{-}\right), \\
U(a, b \mid r) & =\frac{4}{\sqrt{\operatorname{det}\left\|\left(U_{1}+1\right)\left(U_{2}+1\right)\right\|}} \exp \left(-\frac{1}{2} \Pi^{\alpha \beta}\left(U_{1}\right) T_{\alpha \beta}^{+}-\frac{1}{2} \Pi^{\alpha \beta}\left(U_{2}\right) T_{\alpha \beta}^{-}\right),
\end{aligned}
$$

при условии, что $U_{1} U_{2}=U_{r}$ (4.13).

Заметим, что метрика (4.10) инвариантна относительно глобальных левых и правых групповых умножений $S_{\gamma}{ }^{\delta}(X), S \rightarrow H S \widetilde{H}$, где $H$ и $\widetilde{H}$ - некоторые не зависящие от $X$ элементы группы $S p(2)$. Мы воспользуемся этим произволом в разделе 6 при анализе уравнений вне горизонта черной дыры. Для этого выберем

$$
S_{\gamma \delta}=\left(H S_{0}\right)_{\gamma \delta}
$$

с постоянной матрицей $H$ вида

$$
H_{\gamma}{ }^{\delta}=\left(\begin{array}{cc}
\alpha & \beta \\
\beta & \alpha
\end{array}\right)
$$

где $\alpha^{2}=A\left(r_{0}\right)$ и $\beta^{2}=B\left(r_{0}\right)$ в некоторой точке $r_{0}>r_{+}$. Из $(2.10)$ следует, что $\alpha^{2}-\beta^{2}=1$. Новая матрица $S_{\gamma \delta}$ имеет вид

$$
S_{\gamma \delta}=\left(\begin{array}{cc}
\alpha(y-v)+\beta(x-u) & \alpha(x+u)-\beta(y+v) \\
\beta(y-v)+\alpha(x-u) & \beta(x+u)-\alpha(y+v)
\end{array}\right) .
$$

Принимая во внимание (4.11) и (5.7), получаем, что такое переопределение достигается звездочным преобразованием

$$
g\left(a, b \mid W_{1}, W_{2}\right) \rightarrow K(a, b \mid H) \star g\left(a, b \mid W_{1}, W_{2}\right),
$$

где

$$
K(a, b \mid H)=\frac{2}{\sqrt{\operatorname{det}\|H+1\|}} e^{-\Pi^{\gamma \delta}(H) T_{\gamma \delta}^{+} / 2} .
$$

Таким образом, тетрада и лоренцева связность не меняются при преобразовании (5.12).

Заметим, что $K(a, b \mid 1)=1$. В следующем разделе будет показано, что случай $K(a, b \mid H) \neq 1$ играет роль регуляризации, позволяющей анализировать задачу вне точки, где решение сингулярно. Получив решение, мы избавимся от регуляризации, положив $\alpha=1, \beta=0$.

\section{6. РАЗВЕРНУТЫЕ УРАВНЕНИЯ ДЛЯ БЕЗМАССОВЫХ ПОЛЕЙ В ТРЕХМЕРНОМ ПРОСТРАНСТВЕ И МОДУЛЬ ФОКА}

При описании свободных динамических уравнений для безмассовых полей в поле черной дыры БТЗ мы следуем развернутой формулировке, предложенной в работах [28], [22], [20]. В частности, как показано в [22], динамику свободных безмассовых полей спина $s=0$ и $s=1 / 2$ в пространстве AdS можно описать в явно 
конформно-инвариантном виде в терминах сечений некоторого расслоения Фока. А именно, рассмотрим пространственно-временные поля, принимающие значения в образованном осцилляторами $b^{\alpha}$ модуле Фока,

$$
|C(b \mid X)\rangle=C(b \mid X) \star|0\rangle\langle 0|,
$$

где $C(b \mid X)$ - производящая функция

$$
C(b \mid X)=\sum_{k=0}^{\infty} \frac{1}{k !} C_{\alpha_{1} \ldots \alpha_{k}}(X) b^{\alpha_{1}} \ldots b^{\alpha_{k}},
$$

a $|0\rangle\langle 0|=e^{-2 a_{\gamma} b^{\gamma}}-$ фоковский вакуум, удовлетворяющий условиям

$$
a_{\alpha} \star|0\rangle\langle 0|=0, \quad| 0\rangle\langle 0| \star b_{\alpha}=0 .
$$

Динамические безмассовые скаляр и спинор отождествим с низшими компонентами мультиплета:

$$
C(X)=\left.C(b \mid X)\right|_{b=0}, \quad C_{\alpha}(X)=\left.\frac{\partial}{\partial b^{\alpha}} C(b \mid X)\right|_{b=0} .
$$

Тогда динамические уравнения для безмассовых полей в пространстве, локально имеющем геометрию $\mathrm{AdS}_{3}$, можно записать в развернутом виде:

$$
d|C(b \mid X)\rangle-w_{0}(a, b \mid X) \star|C(b \mid X)\rangle=0,
$$

где $w_{0}(a, b \mid X)$ удовлетворяет условию нулевой кривизны (4.2). Покажем, что уравнение (6.5) эквивалентно конформным уравнениям Клейна-Гордона и Дирака и соотношениям, выражающим высшие компоненты мультиплета (6.2) через старшие производные динамических полей [22]. Используя (4.1), уравнение (6.5) можно переписать в виде

$$
D C_{\alpha_{1} \ldots \alpha_{k}}=\frac{k(k-1)}{4} h_{\left(\alpha_{1} \alpha_{2}\right.} C_{\left.\alpha_{3} \ldots \alpha_{k}\right)}+\frac{1}{4} h^{\beta \lambda} C_{\beta \lambda \alpha_{1} \ldots \alpha_{k}}
$$

где скобки в индексах означают полную симметризацию, а $D$ - лоренц-ковариантный дифференциал

$$
D C_{\alpha_{1} \ldots \alpha_{k}}=d C_{\alpha_{1} \ldots \alpha_{k}}+\frac{k}{2} \omega_{\left(\alpha_{1}\right.}^{\gamma} C_{\left.\gamma \alpha_{2} \ldots \alpha_{k}\right)} .
$$

Полагая в $(6.6) k=0$ и $k=2$, имеем

$$
\begin{aligned}
D_{n} C & =\frac{1}{4} h_{n},{ }^{\alpha \beta} C_{\alpha \beta}, \\
D_{n} C_{\alpha \beta} & =\frac{1}{2} h_{n, \alpha \beta} C+\frac{1}{4} h_{n},{ }^{\gamma \delta} C_{\alpha \beta \gamma \delta} .
\end{aligned}
$$

Используя полную симметрию по индексам $C_{\alpha \beta \gamma \delta}$ (т.е. бесследовость), из (6.7) получаем уравнение Клейна-Гордона для скалярного поля $C(X)$

$$
\square C \equiv D^{n} D_{n} C=\frac{3}{4} C .
$$


Аналогично, полагая $k=1$ в уравнении (6.6), получаем уравнение Дирака

$$
h^{n}{ }_{, \alpha \beta} D_{n} C^{\beta}=0 .
$$

Остальные поля мультиплета (6.2) выражаются с помощью (6.6) через производные от физических полей (6.4).

Калибровочное преобразование (4.5) действует на модуле Фока естественным образом:

$$
\delta|C(b \mid X)\rangle=\epsilon(a, b \mid X) \star|C(b \mid X)\rangle .
$$

В частности, преобразование Лоренца калибровочной функции (5.8) действует как

$$
|C(b \mid X)\rangle \rightarrow \Lambda(a, b \mid V) \star\left|C\left(b_{\alpha} \mid X\right)\right\rangle=\left|C\left(V_{\alpha}^{\beta} b_{\beta} \mid X\right)\right\rangle,
$$

где $\Lambda(a, b \mid V)$ определено в (5.9).

Выбирая $w_{0}(a, b \mid X)$ в чисто калибровочном виде $(5.1)$, находим общее локальное решение для $|C(b \mid X)\rangle$ :

$$
|C(b \mid X)\rangle=g^{-1}(a, b \mid X) \star\left|C\left(b \mid X_{0}\right)\right\rangle=g^{-1}(a, b \mid X) \star C(b) \star|0\rangle\langle 0|,
$$

где $\left|C\left(b \mid X_{0}\right)\right\rangle=C(b) \star|0\rangle\langle 0|$ играет роль начальных данных. Смысл формулы (6.12) состоит в следующем: при $g\left(a, b \mid X_{0}\right)=1$ в некоторой точке $X=X_{0}$ она представляет собой ковариантное разложение Тейлора в этой точке, восстанавливающее решение через его производные, параметризованные функцией $C(b)$ на массовой оболочке. Заметим, что данная интерпретация имеет место для любой регулярной точки $X_{0}$, если переопределить калибровочную функцию

$$
g(a, b \mid X) \rightarrow \tilde{g}(a, b \mid X)=g^{-1}\left(a, b \mid X_{0}\right) \star g(a, b \mid X) .
$$

Это изменение не влияет на тетраду (4.7) и связность (4.8), но эффективно переопределяет функцию $C(b)$ :

$$
|C(b \mid X)\rangle=\tilde{g}^{-1}(a, b \mid X) \star \widetilde{C}(b) \star|0\rangle\langle 0|, \quad \widetilde{C}(b) \star| 0\rangle\left\langle 0\left|=g^{-1}\left(a, b \mid X_{0}\right) \star C(b) \star\right| 0\right\rangle\langle 0| .
$$

Очевидно, что данный формализм неприменим в точке $X_{0}$, в которой решение $C(b \mid X)$ сингулярно. На практике наличие пространственно-временной сингулярности в некоторой точке $X_{0}$ эквивалентно отсутствию $\widetilde{C}(b) \star|0\rangle\langle 0|$ (заметим, что звездочное произведение неполиномиальных функций не обязательно хорошо определено). Решение проблемы состоит в некотором переопределении (6.13), которое соответствовало бы анализу в некоторой регулярной точке решения.

Развернутая форма безмассовых уравнений (6.6) явно конформно-инвариантна, причем трехмерная конформная алгебра $s p(4) \sim o(3,2)$ образована различными билинейными комбинациями осцилляторов (3.2). Конструкцию можно обобщить на массивный случай, заменяя обычные осцилляторы $a_{\alpha}, b^{\alpha}$ на деформированные, как это сделано в [13] (см. ссылки там же), или используя модуль Фока, как в [22] и 
в данной статье, но с удвоенным числом деформированных осцилляторов. (Заметим, что в массивном случае в силу свойств деформированных осцилляторов конформная алгебра $s p(4)$, как и положено, нарушается до $\operatorname{AdS}_{3}$-алгебры $s p(2) \oplus s p(2)$.) Соответствующая формулировка технически более сложна, поэтому случай произвольной массы здесь не рассматривается.

В стандартном описании случай массивного скалярного поля в метрике черной дыры БТЗ (2.4) был впервые рассмотрен в [23], [24]. Решение уравнения

$$
\square C=m^{2} C
$$

с определенной энергией $E$ и угловым моментом $L$ имеет вид

$$
C(t, r, \phi)=e^{-i E t} e^{i L \phi} R(r)=\left(1-A(r)^{-1}\right)^{(P+Q) / 2} A(r)^{-\gamma} f(r),
$$

где

$$
P=i \frac{E-L}{2\left(r_{+}-r_{-}\right)}, \quad Q=i \frac{E+L}{2\left(r_{+}+r_{-}\right)}, \quad m^{2}=4 \gamma(1-\gamma)
$$

и

$f(r)=K_{1} F\left(P+\gamma, Q+\gamma, 2 \gamma ; A(r)^{-1}\right)+K_{2} A(r)^{2 \gamma-1} F\left(P+1-\gamma, Q+1-\gamma, 2-2 \gamma ; A(r)^{-1}\right)$,

$K_{1}, K_{2}$ - произвольные константы интегрирования, $F(a, b, c ; x)$ - гипергеометрическая функция, а функция $A(r)$ определена в (2.10). Заметим, что подстановка $\gamma \rightarrow 1-\gamma$ меняет местами два независимых базисных решения. Согласно (6.8) в безмассовом случае $m^{2}=3 / 4$, а значит, $\gamma=1 / 4$.

Ниже будет показано, как воспроизвести в нашем подходе известные результаты для безмассовых скалярного и спинорного полей в метрике черной дыры БТЗ. Для того чтобы выделить состояния с определенной энергией и угловым моментом в мультиплете $|C(b \mid X)\rangle$, наложим следующие условия:

$$
\epsilon_{t} \star|C(b \mid X)\rangle=-i E|C(b \mid X)\rangle, \quad \epsilon_{\phi} \star|C(b \mid X)\rangle=i L|C(b \mid X)\rangle,
$$

где $\epsilon_{t}=g^{-1} \star \xi_{t} \star g, \epsilon_{\phi}=g^{-1} \star \xi_{\phi} \star g-$ генераторы симметрии векторов Киллинга черной дыры БТЗ $\partial / \partial \phi(2.15)$ и $\partial / \partial t(2.16)$. Используя (6.12), перепишем (6.16) в виде

$$
\xi_{t} \star C(b) \star|0\rangle\langle 0|=-i E C(b) \star| 0\rangle\left\langle 0\left|, \quad \xi_{\phi} \star C(b) \star\right| 0\right\rangle\langle 0|=i L C(b) \star| 0\rangle\langle 0| .
$$

Для дальнейшего анализа этих уравнений, определяющих $C(b)$, необходимо знать представление генераторов $\xi_{t}$ и $\xi_{\phi}$ в звездочной алгебре. Оно будет найдено в разделе 7.

Обратим внимание на следующее обстоятельство. Решение (6.14) сингулярно в точке $r=r_{+}$. Следовательно, его нельзя получить в рамках развернутого формализма, используя разложение в окрестности горизонта. Действительно, в разделе 8 будет показано, что уравнения (6.17), отвечающие разложению около $r=r_{+}$, не 
имеют регулярных по осцилляторам $b^{\alpha}$ решений для $C(b)$ и не могут интерпретироваться в терминах модуля Фока. Заметим, что, поскольку калибровочная функция (5.3) регулярна на горизонте, сингулярность в решении следует из условия, что оно имеет определенную энергию и угловой момент, и может отсутствовать, если это условие ослабить.

Чтобы увидеть, что калибровочная функция (5.3) действительно отвечает разложению в окрестности горизонта, переведем ее в единицу, используя преобразование Лоренца. По существу, согласно (5.8) и (6.12) преобразование Лоренца $\Lambda\left(a, b \mid W_{2}\right)$ действует на $g\left(a, b \mid W_{1}, W_{2}\right)$ как

$$
\tilde{g}\left(a, b \mid W_{1}, W_{2}\right)=g\left(a, b \mid W_{1}, W_{2}\right) \star \Lambda^{-1}\left(a, b \mid W_{2}\right)=g\left(a, b \mid W_{1} W_{2}, 1\right),
$$

и, следовательно,

$$
\tilde{g}\left(a, b \mid W_{1}\left(X_{0}\right), W_{2}\left(X_{0}\right)\right)=1, \quad W_{1}\left(X_{0}\right) W_{2}\left(X_{0}\right)=1 .
$$

Выбор калибровочной функции (5.15) с $H_{\gamma}{ }^{\delta}=\delta_{\gamma}{ }^{\delta}$ соответствует $S_{\gamma}{ }^{\delta}=$ $\left(H W_{1} W_{2}\right)_{\gamma}{ }^{\delta}=\delta_{\gamma}{ }^{\delta}$ в точке $X_{0}=\left\{r=r_{+}, t=0, \phi=0\right\}$, принадлежащей горизонту. Действительно, $S_{0}\left(X_{0}\right)_{\gamma}{ }^{\delta}=\delta_{\gamma}{ }^{\delta}$ означает, что $v_{0}=x_{0}=y_{0}=0, u_{0}=1$, что соответствует точке $r_{0}=r_{+}, t_{0}=\phi_{0}=0$. Чтобы избежать этой проблемы, мы используем преобразование (5.15) и получаем (5.14). Теперь $S\left(X_{0}\right)_{\gamma}{ }^{\delta}=\delta_{\gamma}{ }^{\delta}$ в регулярной точке $X_{0}=\left\{r_{0}>r_{+}, t=0, \phi=0\right\}$, кроме случая $\alpha=1, \beta=0$, и возможен совместный развернутый анализ, по крайней мере, в некоторой ее окрестности. Параметрическая регуляризация с $\alpha \neq 1$ и $\beta \neq 0$ необходима для промежуточных вычислений (см. приложение $\mathrm{A}$ ), тогда как предел $\alpha \rightarrow 1, \beta \rightarrow 0$ будет взят в окончательном выражении для $C(b \mid X)$. Напомним, что неопределенность в $H_{\gamma}{ }^{\delta}$ не влияет на связность черной дыры БТЗ (4.19).

\section{7. ЗВЕЗДОЧНАЯ РЕАЛИЗАЦИЯ ВЕКТОРОВ КИЛЛИНГА В ПРОСТРАНСТВЕ $\mathrm{AdS}_{3}$}

Любой вектор Киллинга $\partial / \partial \zeta$ пространства $\mathrm{AdS}_{3}$ является линейной комбинацией генераторов $J_{a b}(2.14)$, т.е.

$$
\frac{\partial}{\partial \zeta}=\Omega^{a b} J_{a b}
$$

где $\Omega^{a b}=-\Omega^{b a}-$ некоторые константы. В звездочной алгебре вектору Киллинга соответствует генератор глобальной симметрии $\xi$, принадлежащий алгебре $s p(2) \oplus$ $s p(2)$, т.е.

$$
\xi=\left(\kappa_{1}\right)^{\alpha \beta} L_{\alpha \beta}+\left(\kappa_{2}\right)^{\alpha \beta} P_{\alpha \beta}
$$

с некоторыми постоянными матрицами $\kappa_{1}$ и $\kappa_{2}$. Чтобы найти $\xi_{t}$ и $\xi_{\phi}$, соответствующие векторам Киллинга метрики БТЗ $\partial / \partial t$ и $\partial / \partial \phi$, вычислим действие генераторов $L_{\alpha \beta}$ и $P_{\alpha \beta}$ на скалярном поле на массовой оболочке. При этом будем использовать калибровочную функцию (5.15) с $S_{\alpha \beta}(5.14)$. 
Введем производящие параметры $\xi^{L}=\left(\kappa_{1}\right)^{\alpha \beta} L_{\alpha \beta}$ для генератора преобразований Лоренца и $\xi^{P}=\left(\kappa_{2}\right)^{\alpha \beta} P_{\alpha \beta}$ для генератора AdS-трансляций. Используя (5.2), (5.3), (6.10) и уравнения движений, нетрудно получить (подробнее см. приложение Б)

$$
\begin{aligned}
& \delta^{L} C(X)=\frac{1}{2}\left(\kappa_{1}\right)^{\alpha \beta} \mathcal{L}_{\alpha \beta}{ }^{n} \partial_{n} C(X), \\
& \delta^{P} C(X)=\left(\kappa_{2}\right)^{\alpha \beta} \mathcal{P}_{\alpha \beta},{ }^{n} \partial_{n} C(X),
\end{aligned}
$$

где

$$
\mathcal{L}_{\alpha \beta, n}=\frac{1}{2}\left(\partial_{n} S_{\alpha \gamma} S_{\beta}^{\gamma}+\partial_{n} S_{\gamma \alpha} S_{\beta}^{\gamma}\right), \quad \mathcal{P}_{\alpha \beta, n}=\partial_{n} S_{\alpha \gamma} S_{\beta}^{\gamma}-\partial_{n} S_{\gamma \alpha} S_{\beta}^{\gamma}
$$

Подставляя (5.14) в (7.4) и сравнивая получившиеся выражения с векторами Киллинга пространства AdS (2.14), имеем

$$
\begin{aligned}
\mathcal{L}_{\gamma \delta} & =\left(\begin{array}{cc}
\alpha \beta\left(J_{12}-J_{03}\right)-\alpha^{2} J_{23}-\beta^{2} J_{01}+J_{02} & -\alpha \beta\left(J_{01}+J_{23}\right)-\alpha^{2} J_{03}+\beta^{2} J_{12} \\
-\alpha \beta\left(J_{01}+J_{23}\right)-\alpha^{2} J_{03}+\beta^{2} J_{12} & \alpha \beta\left(J_{12}-J_{03}\right)-\alpha^{2} J_{23}-\beta^{2} J_{01}-J_{02}
\end{array}\right), \\
\mathcal{P}_{\gamma \delta} & =2\left(\begin{array}{cc}
\alpha \beta\left(J_{12}-J_{03}\right)-\beta^{2} J_{23}-\alpha^{2} J_{01}+J_{13} & -\alpha \beta\left(J_{01}+J_{23}\right)-\beta^{2} J_{03}+\alpha^{2} J_{12} \\
-\alpha \beta\left(J_{01}+J_{23}\right)-\beta^{2} J_{03}+\alpha^{2} J_{12} & \alpha \beta\left(J_{12}-J_{03}\right)-\beta^{2} J_{23}-\alpha^{2} J_{01}-J_{13}
\end{array}\right) .
\end{aligned}
$$

Отсюда следует, что компоненты $J_{03}$ и $J_{12}$, входящие в векторы Киллинга метрики БТ3 (2.15) и (2.16), имеют вид

$$
\begin{aligned}
& J_{03}=-\frac{1}{4} \tau_{1}^{\gamma \delta} \mathcal{P}_{\gamma \delta}-\frac{1}{2} \tau_{2}^{\gamma \delta} \mathcal{L}_{\gamma \delta}, \\
& J_{12}=\frac{1}{4} \tau_{2}^{\gamma \delta} \mathcal{P}_{\gamma \delta}+\frac{1}{2} \tau_{1}^{\gamma \delta} \mathcal{L}_{\gamma \delta},
\end{aligned}
$$

где

$$
\tau_{1}^{\gamma \delta}=\left(\begin{array}{cc}
-\alpha \beta & \beta^{2} \\
\beta^{2} & -\alpha \beta
\end{array}\right), \quad \tau_{2}^{\gamma \delta}=\left(\begin{array}{cc}
-\alpha \beta & \alpha^{2} \\
\alpha^{2} & -\alpha \beta
\end{array}\right) .
$$

Заметим, что матрицы $\tau_{1}$ и $\tau_{2}$ удовлетворяют соотношениям

$$
\begin{gathered}
\frac{1}{2} \tau_{1 \gamma \delta} \tau_{1}^{\gamma \delta}=\beta^{2}, \quad \frac{1}{2} \tau_{2 \gamma \delta} \tau_{2}^{\gamma \delta}=\alpha^{2}, \quad \tau_{1 \gamma \delta} \tau_{2}^{\gamma \delta}=0, \\
\tau_{2}^{\gamma \delta}-\tau_{1}^{\gamma \delta}=\left(\begin{array}{ll}
0 & 1 \\
1 & 0
\end{array}\right) .
\end{gathered}
$$

Таким образом, осцилляторное представление векторов Киллинга метрики БТЗ в реализации модуля Фока имеет вид

$$
\begin{aligned}
\xi_{t} & =\frac{1}{2}\left(r_{-} \tau_{1}^{\gamma \delta}+r_{+} \tau_{2}^{\gamma \delta}\right) L_{\gamma \delta}+\frac{1}{4}\left(r_{+} \tau_{1}^{\gamma \delta}+r_{-} \tau_{2}^{\gamma \delta}\right) P_{\gamma \delta} \\
\xi_{\phi} & =-\frac{1}{2}\left(r_{+} \tau_{1}^{\gamma \delta}+r_{-} \tau_{2}^{\gamma \delta}\right) L_{\gamma \delta}-\frac{1}{4}\left(r_{-} \tau_{1}^{\gamma \delta}+r_{+} \tau_{2}^{\gamma \delta}\right) P_{\gamma \delta}
\end{aligned}
$$




\section{8. ЯВНЫЕ РЕШЕНИЯ ДЛЯ БЕЗМАССОВЫХ ПОЛЕЙ}

Зная осцилляторную реализацию векторов Киллинга метрики БТЗ (7.8), (7.9), перепишем уравнения (6.17) на производящую функцию для поля с определенной энергией и угловым моментом в виде

$$
\begin{aligned}
& \left(\tau_{2}-\tau_{1}\right)^{\gamma \delta}\left(L-\frac{1}{2} P\right)_{\gamma \delta} \star C(b) \star|0\rangle\langle 0|=-4 P C(b) \star| 0\rangle\langle 0|, \\
& \left(\tau_{2}+\tau_{1}\right)^{\gamma \delta}\left(L+\frac{1}{2} P\right)_{\gamma \delta} \star C(b) \star|0\rangle\langle 0|=-4 Q C(b) \star| 0\rangle\langle 0|,
\end{aligned}
$$

где $P$ и $Q$ определены в (6.15). Пусть $b^{\alpha}=(p, q)$. Тогда система (8.1) сводится к двум дифференциальным уравнениям второго порядка

$$
\begin{aligned}
& \left(p \partial_{p}-q \partial_{q}+p q-\partial_{p} \partial_{q}\right) C(p, q)=-4 P C(p, q) \\
& -\alpha \beta\left(\partial_{p} \partial_{p}+\partial_{q} \partial_{q}+p^{2}+q^{2}+2 p \partial_{q}-2 q \partial_{p}\right) C(p, q)+ \\
& \quad+\left(\alpha^{2}+\beta^{2}\right)\left(p \partial_{p}-q \partial_{q}+\partial_{p} \partial_{q}-p q\right) C(p, q)=-4 Q C(p, q) .
\end{aligned}
$$

Заметим, что случай $\alpha=1, \beta=0$ вырожден, поскольку сумма уравнений (8.2) и (8.3) сводится к уравнению первого порядка. В результате система не имеет регулярных по $b^{\alpha}$ решений. Действительно, в этом случае из (8.2) и (8.3) следует, что $\left(p \partial_{p}-q \partial_{q}\right) C(p, q)=-2(P+Q) C(p, q)$, и поэтому функция $C(p, q)=p^{-2(P+Q)} \chi(p q)$ не регулярна по осцилляторам $b^{\alpha}$ для физических значений $P$ и $Q$.

При помощи подстановки $C(p, q)=e^{p q} f(p, q)$ уравнение (8.2) сводится к

$$
\left(\partial_{p} \partial_{q}+2 q \partial_{q}\right) f(p, q)=(4 P-1) f(p, q) .
$$

Его решение можно представить в виде

$$
f(p, q)=\int_{-\infty}^{\infty} e^{-\alpha s^{2} /(4 \beta)} g(s) e^{p s}\left(\frac{s}{2}+q\right)^{2 P-1 / 2} d s,
$$

где $g(s)$ - произвольная функция. Подставляя (8.5) в (8.3), получаем дифференциальное уравнение на $g(s)$

$$
\alpha \beta g^{\prime \prime}(s)-\frac{1}{2} s g^{\prime}(s)-\left(Q+\frac{1}{4}\right) g(s)=0,
$$

которое является вырожденным гипергеометрическим уравнением. Общее решение можно написать в интегральной форме как суперпозицию двух базисных решений

$$
\int_{0}^{\infty} w^{2 Q-1 / 2} e^{-\alpha \beta w^{2}+s w} d w \quad \text { и } \quad \int_{0}^{\infty} w^{2 Q-1 / 2} e^{-\alpha \beta w^{2}-s w} d w .
$$

Интегралы, очевидно, сходятся, поскольку $\alpha \beta>0$ и $\operatorname{Re} Q>-1 / 4$.

Обозначим общее решение уравнения (8.6)

$$
\int w^{2 Q-1 / 2} e^{-\alpha \beta w^{2}+s w} d w
$$


понимая под этим линейную комбинацию интегралов

$$
\int_{0}^{\infty} w^{2 Q-1 / 2} e^{-\alpha \beta w^{2}+s w} d w \quad \text { и } \quad \int_{-\infty}^{0} w^{2 Q-1 / 2} e^{-\alpha \beta w^{2}+s w} d w .
$$

Заметим, что несмотря на многозначность второго интеграла в (8.9) неопределенность, выражающаяся в виде произвольного постоянного фазового множителя, всегда может быть включена в константу интегрирования.

Используя $g(s)$ из (8.8) и делая замену переменной интегрирования $s \rightarrow s-2 q$ в (8.5), получаем производящую функцию в виде

$$
\begin{aligned}
C(p, q) & =e^{-p q} \int_{-\infty}^{\infty} d s \int d w e^{-\alpha(s-2 q)^{2} /(4 \beta)-\alpha \beta w^{2}+(s-2 q) w+s p} s^{2 P-1 / 2} w^{2 Q-1 / 2}= \\
& =\int_{-\infty}^{\infty} d s \int d w e^{m_{\gamma \delta} b^{\gamma} b^{\delta}+n_{\gamma} b^{\gamma}} e^{-\alpha \beta w^{2}+s w-\alpha s^{2} /(4 \beta)} s^{2 P-1 / 2} w^{2 Q-1 / 2},
\end{aligned}
$$

где

$$
m_{\gamma \delta}=\left(\begin{array}{cc}
0 & -1 / 2 \\
-1 / 2 & -\alpha / \beta
\end{array}\right), \quad n_{\gamma}=\left(s, \frac{\alpha}{\beta} s-2 w\right) .
$$

Используя (8.10) и (5.15), вычислим производящую функцию (6.12), переопределив переменную интегрирования $\beta w \rightarrow w$ и положив в конце $\alpha=1, \beta=0$. С точностью до постоянного множителя имеем следующее интегральное представление для производящей функции $C(b \mid X)$ (см. приложение $\mathrm{A})$ :

$$
\begin{aligned}
& C(b \mid t, r, \phi)=e^{-i E t} e^{i L \phi} A(r)^{-Q-1 / 2}\left(1-(A(r))^{-1}\right)^{(P+Q) / 2} \times \\
& \times e^{-b^{1} b^{2}} \int_{-\infty}^{\infty} d s \int d w s^{2 P-1 / 2} w^{2 Q-1 / 2} \times \\
& \times \exp \left(-\frac{s^{2}}{4}-\frac{w^{2}}{4 A(r)}+\frac{s w}{2 A(r)}+\mu(r) s b^{1}-\eta(r) w b^{2}\right),
\end{aligned}
$$

где $A(r)$ и $\mu(r), \eta(r)$ определены в (2.10) и (4.17), соответственно. Заметим, что, как уже обсуждалось в разделе 6 и в начале этого раздела, данный формализм не позволяет положить $\alpha=1, \beta=0$ в $C(b)$ до выполнения звездочного умножения c $g^{-1}\left(a, b \mid W_{1}, W_{2}\right)$.

По построению производящая функция (8.11) содержит решения для безмассовых полей в метрике черной дыры БТЗ вместе со всеми их производными на массовой оболочке в виде коэффициентов разложения по степеням осцилляторов $b^{\alpha}$. Используя стандартное интегральное представление для гипергеометрической функ- 
ции (см., например, [29]), перепишем производящую функцию (8.11) в виде

$$
\begin{aligned}
& C(b \mid t, r, \phi)=e^{-i E t} e^{i L \phi}\left(1-A^{-1}\right)^{(P+Q) / 2} A^{-1 / 4} \times \\
& \times \sum_{m=0}^{\infty} \sum_{n=0}^{\infty} \frac{\mu^{m}(-\eta)^{n}}{m ! n !} A^{n / 2}\left(b^{1}\right)^{m}\left(b^{2}\right)^{n} e^{-b^{1} b^{2}} \times \\
& \times\left[K_{1} F\left(P+\frac{2 m+1}{4}, Q+\frac{2 n+1}{4}, \frac{1}{2} ; A^{-1}\right)+\right. \\
&\left.+K_{2} A^{-1 / 2} F\left(P+\frac{2 m+3}{4}, Q+\frac{2 n+3}{4}, \frac{3}{2} ; A^{-1}\right)\right],
\end{aligned}
$$

где $K_{1}, K_{2}$ - произвольные константы интегрирования, а $P, Q$ определены в (6.15).

Как уже было сказано в разделе 6 , скалярное поле описывается функцией $C(0 \mid X)$ (6.4). Таким образом, из (8.12) имеем

$$
\begin{aligned}
& C(t, r, \phi)=e^{-i E t} e^{i L \phi}\left(1-A^{-1}\right)^{(P+Q) / 2} A^{-1 / 4} \times \\
& \quad \times\left[K_{1} F\left(P+\frac{1}{4}, Q+\frac{1}{4}, \frac{1}{2} ; A^{-1}\right)+K_{2} A^{-1 / 2} F\left(P+\frac{3}{4}, Q+\frac{3}{4}, \frac{3}{2} ; A^{-1}\right)\right] .
\end{aligned}
$$

Этот результат совпадает с решением уравнения (6.8) для безмассового скаляра в метрике черной дыры БТЗ, первоначально найденным в [23], [24] для поля произвольной массы.

Аналогично из (8.12) сразу получаем решение для спинорного поля $C_{\alpha}(X)(6.4)$ :

$$
C_{\alpha}(t, r, \phi)=e^{-i E t} e^{i L \phi}\left(1-A^{-1}\right)^{(P+Q) / 2} A^{-1 / 4}\left(K_{1} \psi_{1 \alpha}+K_{2} \psi_{2 \alpha}\right),
$$

где

$$
\begin{aligned}
& \psi_{1}=\left(\begin{array}{c}
\mu F\left(P+3 / 4, Q+1 / 4,1 / 2 ; A^{-1}\right) \\
-(Q+1 / 4) \eta F\left(P+3 / 4, Q+5 / 4,3 / 2 ; A^{-1}\right)
\end{array}\right), \\
& \psi_{2}=\left(\begin{array}{c}
(P+1 / 4) \mu A^{-1 / 2} F\left(P+5 / 4, Q+3 / 4,3 / 2 ; A^{-1}\right) \\
-\eta A^{1 / 2} F\left(P+1 / 4, Q+3 / 4,1 / 2 ; A^{-1}\right)
\end{array}\right) .
\end{aligned}
$$

Различный выбор функций $\mu(r), \eta(r)$ (4.17) параметризует различные лоренцевы калибровки в общем решении уравнения Дирака (6.9) с определенной энергией $E$ и угловым моментом $L$ в метрике черной дыры БТЗ. Заметим, что наша калибровка отличается от метрики, используемой в работах [25], [26].

\section{9. ЭКСТРЕМАЛЬНАЯ ЧЕРНАЯ ДЫРА БТЗ}

Точные решения уравнений Клейна-Гордона и Дирака в поле экстремальной черной дыры БТЗ были впервые найдены в работах [30] и [31]. В экстремальном случае $(M=|J|)$ оба горизонта совпадают и уже нельзя пользоваться параметризацией (2.9). Как и прежде, связность БТЗ $w_{0}(a, b \mid X)$ выражается через калибровочную функцию $g\left(a, b \mid W_{1}, W_{2}\right)$, но теперь координаты $X^{a}$ параметризованы 
иначе (см. [11]). В экстремальном случае вектор Киллинга, отвечающий за факторизацию (2.13), содержит дополнительные члены, от которых нельзя избавиться $S O(2,2)$-преобразованиями,

$$
\frac{\partial}{\partial \phi}=-\lambda r_{+} J_{12}+\lambda r_{-} J_{03}+J_{13}-J_{23}
$$

Следовательно, система уравнений (8.1) имеет другой вид. К счастью, для того чтобы получить решения в экстремальном случае, не нужно решать эти уравнения снова. Как было отмечено, например, в [32], можно просто перейти к пределу в общих решениях (8.13) и (8.14). А именно, произведение

$$
A^{-1} P=\kappa=i \frac{(E-L)\left(r_{+}+r_{-}\right)}{2\left(r^{2}-r_{-}^{2}\right)}
$$

регулярно в пределе $r_{+} \rightarrow r_{-}\left(P\right.$ определено в (6.15)). Подставим теперь $A^{-1}=$ $\kappa / P$ в $(8.13),(8.14)$ и рассмотрим предел $r_{+} \rightarrow r_{-}$или, другими словами, $P \rightarrow \infty$. Результат может быть представлен в терминах функций Уиттекера $M_{p, q}(x)$ [33].

Для безмассового скаляра имеем

$$
C(t, r, \phi)=e^{-i E t} e^{i L \phi}\left(K_{1} M_{-Q,-1 / 4}\left(\kappa_{\mathrm{e}}\right)+K_{2} M_{-Q, 1 / 4}\left(\kappa_{\mathrm{e}}\right)\right),
$$

где

$$
\kappa_{\mathrm{e}}=i \frac{(E-L) r_{\mathrm{e}}}{r^{2}-r_{\mathrm{e}}^{2}}
$$

и $r_{\mathrm{e}}$ - горизонт экстремальной черной дыры БТЗ. Легко убедиться, что это решение действительно удовлетворяет конформному уравнению Клейна-Гордона, записанному в метрике экстремальной черной дыры.

Для безмассового спинора имеем

$$
C_{\alpha}(t, r, \phi)=e^{-i E t} e^{i L \phi}\left(K_{1} \psi_{1 \alpha}+K_{2} \psi_{2 \alpha}\right),
$$

где

$$
\psi_{1}=\left(\begin{array}{c}
\mu M_{-Q,-1 / 4}\left(\kappa_{\mathrm{e}}\right) \\
-(Q+1 / 4) \eta \kappa_{\mathrm{e}}^{-1 / 2} M_{-Q, 1 / 4}\left(\kappa_{\mathrm{e}}\right)
\end{array}\right), \quad \psi_{2}=\left(\begin{array}{c}
\mu M_{-Q, 1 / 4}\left(\kappa_{\mathrm{e}}\right) \\
-\eta \kappa_{\mathrm{e}}^{-1 / 2} M_{-Q,-1 / 4}\left(\kappa_{\mathrm{e}}\right)
\end{array}\right),
$$

а $K_{1}, K_{2}-$ произвольные константы.

\section{0. СИММЕТРИИ БЕЗМАССОВЫХ ПОЛЕЙ В МЕТРИКЕ ЧЕРНОЙ ДЫРЫ БТЗ}

Любое вакуумное решение (4.1) уравнения (4.2) нарушает локальную симметрию высших спинов до глобальной, порождаемой подалгеброй стабильности с параметром $\epsilon_{0}(a, b \mid X)$, удовлетворяющим уравнению (4.6). Граничные условия БТ3 (2.13) сужают пространство решений уравнения (4.6), представляя собой, таким образом,

2 Теоретическая и математическая физика, т. 153, № 2, 2007 г. 
(нелокальный) механизм спонтанного нарушения симметрии. Другими словами, только те симметрии остаются глобально определенными при факторизации (2.13), которые коммутируют с вектором Киллинга $\xi_{\phi}$, отвечающим за отождествление,

$$
\left[\xi(a, b), \xi_{\phi}\right]_{\star}=0,
$$

где $\xi(a, b)$ - производящий параметр в уравнении (5.2). Пространства решений уравнения (10.1) различны для общего случая и случая экстремальной черной дыры. Поэтому рассмотрим их независимо.

Начнем с общего случая $r_{+}^{2}-r_{-}^{2}>0$ и вектора Киллинга $\partial / \partial \phi$, заданного в $(2.15)$ и имеющего звездочную реализацию $\xi_{\phi}(7.9)$. Для согласования результатов с (5.3) положим $\alpha=1$ и $\beta=0$ так, что

$$
\xi_{\phi}=-\frac{1}{2} r_{-} \tau^{\gamma \delta} L_{\gamma \delta}-\frac{1}{4} r_{+} \tau^{\gamma \delta} P_{\gamma \delta}, \quad \tau^{\gamma \delta}=\left(\begin{array}{ll}
0 & 1 \\
1 & 0
\end{array}\right) .
$$

При решении уравнения (10.1) удобнее перейти к новому базису осцилляторов $p_{\alpha}, q_{\beta}$ :

$$
\begin{array}{ll}
p_{1}=\frac{1}{\sqrt{2}}\left(a_{1}+b_{1}\right), & p_{2}=\frac{1}{\sqrt{2}}\left(a_{1}-b_{1}\right), \\
q_{1}=\frac{1}{\sqrt{2}}\left(a_{2}-b_{2}\right), & q_{2}=\frac{1}{\sqrt{2}}\left(a_{2}+b_{2}\right),
\end{array}
$$

удовлетворяющих коммутационным соотношениям

$$
\left[p_{\alpha}, p_{\beta}\right]_{\star}=\left[q^{\alpha}, q^{\beta}\right]_{\star}=0, \quad\left[p_{\alpha}, q^{\beta}\right]_{\star}=\delta_{\alpha}{ }^{\beta},
$$

так что параметр $\xi_{\phi}$ имеет следующий простой вид:

$$
\xi_{\phi}=-\frac{1}{2} A_{\alpha}{ }^{\beta} p_{\beta} q^{\alpha}, \quad A_{\alpha}{ }^{\beta}=\left(\begin{array}{cc}
r_{+}+r_{-} & 0 \\
0 & r_{-}-r_{+}
\end{array}\right) .
$$

Заметим, что, поскольку коммутационные соотношения не изменились, как и прежде, для звездочного произведения можно использовать формулу (3.4) с заменой $a$ и $b$ на $p$ и $q$, соответственно.

Из уравнения (10.1) следует (принимая во внимание (А.1))

$$
A_{\alpha}{ }^{\beta}\left(p_{\beta} \frac{\partial}{\partial p_{\alpha}}+q^{\alpha} \frac{\partial}{\partial q^{\beta}}\right) \xi(p, q)=0 .
$$

Искомые инфинитезимальные симметрии высших спинов соответствуют локальным преобразованиям с конечным числом пространственно-временных производных. Соответствующие производящие параметры симметрии $\xi(p, q)$ описываются полиномиальными по осцилляторам функциями. Класс полиномиальных решений уравнения (10.1) характеризуется параметром

$$
\sigma=\frac{r_{+}+r_{-}}{r_{+}-r_{-}}
$$


Имеем следующие случаи.

1. $\sigma \notin \mathbb{N}$. Для любого положительного нецелого $\sigma$ общее решение уравнения (10.6) имеет вид

$$
\xi(p, q)=\sum R_{m n}\left(q_{1} p_{2}\right)^{m}\left(q_{2} p_{1}\right)^{n} \sim \sum \widetilde{R}_{m n}\left(\xi_{\phi}\right)^{m}\left(\xi_{t}\right)^{n},
$$

где $R_{m n}$ - произвольные постоянные. Заметим, что конформная алгебра $s p(4)$, заданная различными билинейными комбинациями осцилляторов (10.3), нарушена до $u(1) \oplus u(1)$-подалгебры, состоящей из векторов Киллинга черной дыры БТЗ $\xi_{\phi}$ и $\xi_{t}$ (эквивалентно $q_{1} p_{2}$ и $q_{2} p_{1}$ ).

2. $\sigma=2,3, \ldots$. В случае положительных целых $\sigma$ сохраняется более широкий класс симметрий высших спинов. Общее решение уравнения (10.6) имеет вид

$$
\xi(p, q)=\sum R_{n_{1} n_{2} m_{1} m_{2}}\left(q_{1} p_{2}\right)^{n_{1}}\left(q_{2} p_{1}\right)^{n_{2}}\left(p_{1} p_{2}^{\sigma}\right)^{m_{1}}\left(q_{1} q_{2}^{\sigma}\right)^{m_{2}} .
$$

Конформная алгебра $s p(4)$ по-прежнему нарушена до $u(1) \oplus u(1)$. Условие $\sigma=$ $2,3, \ldots$ имеет вид некоторого квантования массы $M$ в терминах углового момента $J$, поскольку $\sigma=\sqrt{(M+J \lambda) /(M-J \lambda)}$. В этом случае $\rho^{+}=\left(\rho^{-}\right)^{\sigma}$, т.е. один из операторов голономии, участвующих в факторизации пространства AdS, является целой степенью другого ${ }^{5)}$.

3. $\sigma=1$. Это случай невращающейся черной дыры $J=0$. Полиномиальные решения для $\xi(p, q)$ имеют вид

$$
\xi(p, q)=\sum R_{m_{1} m_{2} n_{1} n_{2}}\left(q_{1} p_{2}\right)^{m_{1}}\left(q_{2} p_{1}\right)^{m_{2}}\left(p_{1} p_{2}\right)^{n_{1}}\left(q_{1} q_{2}\right)^{n_{2}} .
$$

Этот случай выделен тем, что сохраняется большая часть конформных симметрий. Остаточные симметрии порождены билинейными комбинациями вида $q_{1} p_{2}$, $q_{2} p_{1}, p_{1} p_{2}, q_{1} q_{2}$, что изоморфно алгебре $g l(2)$. Помимо векторов Киллинга черной дыры БТЗ она содержит генераторы специальных конформных преобразований, порожденных $b_{1} b_{1}$ и $b_{2} b_{2}$.

Рассмотрим теперь экстремальный случай. Вектор Киллинга экстремальной черной дыры $\left(r_{-}=r_{+}=r_{\mathrm{e}}\right)$, отвечающий за факторизацию, приведен в (9.1). Используя (7.5) и снова полагая $\alpha=1, \beta=0$, имеем для $\xi_{\phi}$ в терминах осцилляторов $p, q$

$$
\xi_{\phi}=-r_{\mathrm{e}} p_{1} q_{2}+\frac{1}{2}\left(p_{1}^{2}-q_{1}^{2}\right) .
$$

Выполнив простые звездочные вычисления, перепишем (10.1) в виде

$$
r_{\mathrm{e}}\left(p^{2} \frac{\partial}{\partial p^{2}}-q^{1} \frac{\partial}{\partial q^{1}}\right) \xi-p^{2} \frac{\partial \xi}{\partial q^{1}}+q^{2} \frac{\partial \xi}{\partial p^{1}}=0 .
$$

Случаи $r_{\mathrm{e}} \neq 0$ и $r_{\mathrm{e}}=0$ (т.е. $M=J=0$ ) требуют отдельного рассмотрения.

А. Случай $r_{\mathrm{e}} \neq 0$. Выпишем общее решение уравнения (10.12) в классе полиномов

$$
\xi(p, q)=\sum R_{m n}\left(p_{1}\right)^{m}\left(2 r_{\mathrm{e}} q_{2}-p_{1}\right)^{m}\left(q_{1}\right)^{n} .
$$

\footnotetext{
5) Мы признательны С. Карлипу, обратившему наше внимание на этот факт.
} 
Заметим, что помимо стандартной $u(1) \oplus u(1)$-симметрии, порожденной векторами Киллинга $\xi_{t}$ и $\xi_{\phi}$, экстремальная черная дыра имеет один спинор Киллинга, порожденный $q_{1}$, что согласуется с результатами работы [34], где была обнаружена суперсимметрия экстремальной черной дыры.

Б. Случай $r_{\mathrm{e}}=0$. В случае "вакуумной" черной дыры $M=J=0$ получаем максимальное число суперсимметрий и общее решение для $\xi(p, q)$ вида

$$
\xi(p, q)=\sum R_{m n k}\left(p_{1}\right)^{m}\left(q_{1}\right)^{n}\left(q_{1} q_{2}+p_{1} p_{2}\right)^{k}
$$

Имеются две суперсимметрии [34], порожденные $p_{1}$ и $q_{1}$, а подалгебра конформной алгебры с генераторами $p_{1} p_{1}, q_{1} q_{1}, q_{1} p_{1}, q_{1} q_{2}+p_{1} p_{2}$ изоморфна $E_{2} \oplus u(1)$, где $E_{2}-$ алгебра движений двумерной евклидовой плоскости.

Заметим, что в нашем подходе формулы законов преобразований симметрий находятся элементарно. Для любого производящего параметра $\xi(a, b)$ соответствующий генератор симметрии (5.2) получается в результате дифференцирования производящего параметра (Б.1) (см. приложение Б) по источникам $\mu_{\alpha}, \eta_{\beta}$.

\section{1. ЗАКЛЮЧЕНИЕ}

В данной работе показано, что черная дыра БТЗ может быть точно описана в рамках формализма звездочной алгебры, лежащей в основе современной нелинейной калибровочной теории высших спинов. Удовлетворяя условию нулевой кривизны в алгебре $o(2,1) \oplus o(2,1)$, черная дыра БТЗ тем самым оказывается точным решением нелинейной теории высших спинов в трех измерениях. Также показано, как данный формализм позволяет решать свободные полевые уравнения в метрике черной дыры.

Найдены остаточные высшие и низшие симметрии безмассовых полей в поле черной дыры БТЗ. В случае неэкстремальной черной дыры БТЗ $(M>0)$ конформная алгебра $o(3,2) \sim s p(4)$ оказывается нарушенной до $u(1) \oplus u(1)$-подалгебры, построенной из векторов Киллинга черной дыры БТЗ, в случае $J>0$ и до $g l(2)$ в случае $J=0$. При $\sigma=\sqrt{(M+J \lambda) /(M-J \lambda)}=1,2, \ldots$ остаточных симметрий высших спинов становится больше. У нас нет какой-либо физической интерпретации этого явления. Анализ экстремальной черной дыры воспроизводит уже известные результаты, касающиеся структуры низших (супер)симметрий, и определяет остаточные симметрии высших спинов.

Мы надеемся развить полученные результаты в двух, вероятно, взаимосвязанных направлениях: получить, во-первых, решения типа Керра в нелинейной теории высших спинов в четырех измерениях и выше, а во-вторых, решения типа БТЗ в обобщенных пространственно-временны́х геометриях с матричными координатами, являющихся $S p(M)$-групповыми многообразиями в AdS-подобном случае. 
ПРИЛОЖЕНИЕ А

\section{Вычисление звездочного произведения}

Используя (3.4), легко получить следующие полезные формулы, которые были использованы нами в статье при вычислении звездочного произведения:

$$
\begin{aligned}
& a_{\alpha} a_{\beta} \star f(a, b)=a_{\alpha} a_{\beta} f(a, b)+\frac{1}{2}\left(a_{\alpha} \frac{\partial}{\partial b^{\beta}}+a_{\beta} \frac{\partial}{\partial b^{\alpha}}\right) f(a, b)+\frac{1}{4} \frac{\partial^{2}}{\partial b^{\alpha} \partial b^{\beta}} f(a, b), \\
& b_{\alpha} b_{\beta} \star f(a, b)=b_{\alpha} b_{\beta} f(a, b)+\frac{1}{2}\left(b_{\alpha} \frac{\partial}{\partial a^{\beta}}+b_{\beta} \frac{\partial}{\partial a^{\alpha}}\right) f(a, b)+\frac{1}{4} \frac{\partial^{2}}{\partial a^{\alpha} \partial a^{\beta}} f(a, b), \\
& a_{\alpha} b_{\beta} \star f(a, b)=a_{\alpha} b_{\beta} f(a, b)+\frac{1}{2}\left(a_{\alpha} \frac{\partial}{\partial a^{\beta}}+b_{\beta} \frac{\partial}{\partial b^{\alpha}}\right) f(a, b)+\frac{1}{4} \frac{\partial^{2}}{\partial a^{\beta} \partial b^{\alpha}} f(a, b) .
\end{aligned}
$$

Вычислим производящую функцию (8.11). Согласно схеме, описанной в разделе 6 ,

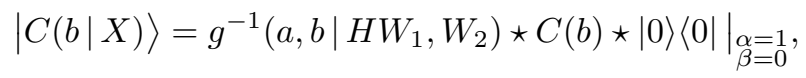

где функция $C(b)$ определена в (8.10). Прямое вычисление довольно громоздкое. Для его упрощения избавимся от лоренцева члена в калибровочной функции $g\left(a, b \mid H W_{1}, W_{2}\right)$. А именно, с помощью (5.7) и (5.8) можно переписать (А.2) в виде

$$
|C(b \mid X)\rangle=\Lambda^{-1}(a, b \mid V) \star g^{-1}\left(a, b \mid H W_{1} V, V^{-1} W_{2}\right) \star C(b) \star|0\rangle\left\langle 0||_{\substack{\alpha=1 \\ \beta=0}} .\right.
$$

Выберем матрицу преобразования Лоренца $V_{\gamma \delta}$ так, что $H W_{1} V=V^{-1} W_{2}=\sqrt{S}$. Тогда калибровочная функция примет вид

$$
g_{0}(a, b \mid S)=g(a, b \mid \sqrt{S}, \sqrt{S})=\frac{4}{\operatorname{det}\|\sqrt{S}+1\|} \exp \left(-\Pi^{\alpha \beta}(\sqrt{S})\left(a_{\alpha} a_{\beta}+b_{\alpha} b_{\beta}\right)\right),
$$

где матрица $S_{\gamma \delta}$ определена в (5.14). Таким образом, используя $(6.11)$, перепишем производящую функцию как

$$
\left|C\left(b_{\gamma} \mid X\right)\right\rangle=\left|C_{0}\left(\left(V_{0}^{-1}\right)_{\gamma}^{\delta} b_{\delta} \mid X\right)\right\rangle
$$

где $V_{0}$ - матрица преобразования Лоренца, вычисленная при $\alpha=1, \beta=0$, имеет следующий вид:

$$
V_{0 \gamma \delta}=-\sqrt{\frac{(u+x)(y-v)}{2(u+1)}}\left(\begin{array}{cc}
\mu(r) & \mu(r) \frac{x-u-1}{y-v} \\
\eta(r)(u-x) & \eta(r) \frac{(u-x)(u+x+1)}{y-v}
\end{array}\right),
$$

a

$$
\left|C_{0}(b \mid X)\right\rangle=g_{0}^{-1}(a, b \mid S) \star C(b) \star|0\rangle\left\langle 0||_{\substack{\alpha=1 \\ \beta=0}} .\right.
$$

Для звездочного умножения гауссовых экспонент вида

$$
F(b) \star|0\rangle\left\langle 0\left|=\sqrt{\operatorname{det}\left\|1-f^{2}\right\|} e^{f^{\alpha \beta}\left(a_{\alpha} a_{\beta}+b_{\alpha} b_{\beta}\right)} \star e^{m_{\gamma \delta} b^{\gamma} b^{\delta}+n_{\gamma} b^{\gamma}} \star\right| 0\right\rangle\langle 0|,
$$


используя (3.4), после простого гауссового интегрирования получаем

$$
\begin{gathered}
F(b)=\sqrt{\frac{\operatorname{det}\left\|1-f^{2}\right\|}{\operatorname{det} A}} \exp \left\{\left[\left(f^{-1}+2 m\right) \frac{1}{f+f^{-1}+4 m}(f+2 m)-m\right]_{\alpha \beta} b^{\alpha} b^{\beta}+\right. \\
\left.+\left[\frac{1}{f+f^{-1}+4 m}\left(f^{-1}-f\right)\right]_{\alpha \beta} n^{\alpha} b^{\beta}+\left[\frac{1}{f+f^{-1}+4 m}\right]_{\alpha \beta} n^{\alpha} n^{\beta}\right\},
\end{gathered}
$$

где $\left(f^{-1}\right)_{\alpha}{ }^{\beta} f_{\beta}^{\gamma}=\delta_{\alpha}^{\gamma}, A_{\alpha \beta}=\varepsilon_{\alpha \beta}+f_{\alpha}^{\gamma} f_{\gamma \beta}+4 f_{\alpha}^{\gamma} m_{\gamma \beta}$. Тогда с учетом (8.10), (А.4) и (А.8) имеем

$$
\begin{aligned}
C_{0}(b \mid X, \alpha, \beta)= & \left(\frac{\beta}{y-v}\right)^{1 / 2} \int_{-\infty}^{\infty} d s \int d w s^{2 P-1 / 2} w^{2 Q-1 / 2} \times \\
& \times \exp \left(-\frac{u+x}{4(y-v)} s^{2}-\beta^{2} \frac{u-x}{y-v} w^{2}+\frac{\beta}{y-v} s w\right) \times \\
& \times \exp \left(B_{\gamma \delta} b^{\gamma} b^{\delta}+\frac{A_{\gamma} b^{\gamma}}{(y-v) \sqrt{2(\alpha u-\beta y+1)}}\right),
\end{aligned}
$$

где

$$
\begin{gathered}
A_{\gamma}=\left(\begin{array}{c}
2 \beta(\alpha(y-v)+\beta(x-u)) w+(\beta+y-v) s \\
2 \beta(\alpha(x-u)+\beta(y-v)-1) w+(u+x+\alpha) s
\end{array}\right), \\
B_{\gamma \delta}=\frac{\beta}{y-v} m_{\gamma \delta}+\frac{y-v+\beta}{2(y-v)(\alpha u-\beta y+1)} S_{(\gamma \delta)},
\end{gathered}
$$

а скобки в индексах означают симметризацию по индексам.

Делая замену переменной интегрирования $\beta w \rightarrow w$ (зависящий от $\beta$ фактор включаем в константу интегрирования) и полагая после этого $\alpha=1, \beta=0$, получаем

$$
\begin{aligned}
C_{0}(b \mid X)=(y & -v)^{-1 / 2} \int_{-\infty}^{\infty} d s \int d w s^{2 P-1 / 2} w^{2 Q-1 / 2} \times \\
& \times \exp \left(-\frac{u+x}{4(y-v)} s^{2}-\frac{u-x}{y-v} w^{2}+\frac{1}{y-v} s w\right) \exp \left(\widehat{B}_{\gamma \delta} b^{\gamma} b^{\delta}\right) \times \\
& \times \exp \left(\frac{b^{1}(2 w+s)(y-v)+b^{2}(s(u+x+1)+2 w(x-u-1))}{(y-v) \sqrt{2(u+1)}}\right),
\end{aligned}
$$

где

$$
\widehat{B}_{\gamma \delta}=\frac{1}{2}\left(\begin{array}{cc}
\frac{y-v}{u+1} & \frac{x}{u+1} \\
\frac{x}{u+1} & -\frac{y+v}{u+1}-\frac{2}{y-v}
\end{array}\right) .
$$

Производя преобразование Лоренца (А.5) и используя БТЗ-координаты (2.9), получаем (8.11).

Отметим также удобную параметризацию для $S p(2)$-матрицы $S_{\alpha \beta}$

$$
S_{\alpha \beta}=\operatorname{ch}(p) \varepsilon_{\alpha \beta}+\operatorname{sh}(p) \kappa_{\alpha \beta},
$$

где $\kappa_{\alpha \beta}=\kappa_{\beta \alpha}$ и $\kappa_{\alpha \beta} \kappa^{\alpha \beta} / 2=-1$. Легко видеть, что $n$-я степень $S$ равна

$$
\left(S^{n}\right)_{\alpha \beta}=\operatorname{ch}(n p) \epsilon_{\alpha \beta}+\operatorname{sh}(n p) \kappa_{\alpha \beta} .
$$


Следовательно,

$$
(\sqrt{S})_{\alpha \beta}=\operatorname{ch}\left(\frac{p}{2}\right) \varepsilon_{\alpha \beta}+\operatorname{sh}\left(\frac{p}{2}\right) \kappa_{\alpha \beta} .
$$

Кроме того, для матрицы $\Pi=(\sqrt{S}-1) /(\sqrt{S}+1)$ имеем

$$
\Pi_{\alpha \beta}=\operatorname{th}\left(\frac{p}{4}\right) \kappa_{\alpha \beta} \text {. }
$$

ПРИЛОЖКНИЕ Б

\section{Действие оператора (момента) импульса на скалярное поле}

Для того чтобы найти действие генераторов $L_{\alpha \beta}$ и $P_{\alpha \beta}$ на скалярное поле на массовой оболочке, рассмотрим производящий параметр вида

$$
\xi=\exp \left(a_{\alpha} \mu^{\alpha}+b_{\alpha} \eta^{\alpha}\right)
$$

с постоянными источниками $\mu_{\alpha}$ и $\eta_{\alpha}$. Как показано в [20], генераторы глобальных симметрий получаются из (5.2)

$$
\epsilon=\exp \left(a_{\alpha} \hat{\mu}^{\alpha}+b_{\alpha} \hat{\eta}^{\alpha}\right)
$$

где

$$
\begin{aligned}
& \hat{\mu}_{\alpha}=\frac{1}{2}\left(W_{1}^{-1}+W_{2}\right)_{\alpha}{ }^{\beta} \mu_{\beta}+\frac{1}{2}\left(W_{1}^{-1}-W_{2}\right)_{\alpha}{ }^{\beta} \eta_{\beta}, \\
& \hat{\eta}_{\alpha}=\frac{1}{2}\left(W_{1}^{-1}+W_{2}\right)_{\alpha}{ }^{\beta} \eta_{\beta}+\frac{1}{2}\left(W_{1}^{-1}-W_{2}\right)_{\alpha}{ }^{\beta} \mu_{\beta} .
\end{aligned}
$$

Дифференцирование по источникам $\mu_{\alpha}, \eta_{\alpha}$ дает генераторы глобальных симметрий пространства $\mathrm{AdS}_{3}$

$$
\begin{aligned}
\epsilon_{\alpha \beta}^{P} & =\left.\left(\frac{\partial^{2}}{\partial \mu^{\alpha} \partial \mu^{\beta}}+\frac{\partial^{2}}{\partial \eta^{\alpha} \partial \eta^{\beta}}\right) \epsilon\right|_{\substack{\mu=0 \\
\eta=0}}, \\
\epsilon_{\alpha \beta}^{L} & =\left.\frac{1}{2}\left(\frac{\partial^{2}}{\partial \mu^{\alpha} \partial \eta^{\beta}}+\frac{\partial^{2}}{\partial \eta^{\alpha} \partial \mu^{\beta}}\right) \epsilon\right|_{\substack{\mu=0 \\
\eta=0}} .
\end{aligned}
$$

Используя (6.10) и выполняя звездочное умножение, получаем

$$
\delta|C(b \mid X)\rangle=\epsilon \star|C(b \mid X)\rangle=\exp \left(b_{\alpha} \hat{\eta}^{\alpha}+\frac{1}{2} \hat{\mu}_{\alpha} \hat{\eta}^{\alpha}\right) C(b+\hat{\mu} \mid X) \star|0\rangle\langle 0|,
$$

откуда

$$
\delta C(b \mid X)=C(b+\hat{\mu} \mid X) \exp \left(b_{\alpha} \hat{\eta}^{\alpha}+\frac{1}{2} \hat{\mu}_{\alpha} \hat{\eta}^{\alpha}\right) .
$$

В результате из (Б.3) и (Б.5) получаем следующее действие AdS-изометрий на скалярном поле $C(X)$ :

$$
\begin{aligned}
\delta_{\alpha \beta}^{P} C(X) & =\left.\left(\frac{\partial^{2}}{\partial \mu^{\alpha} \partial \mu^{\beta}}+\frac{\partial^{2}}{\partial \eta^{\alpha} \partial \eta^{\beta}}\right)\left(C(\hat{\mu} \mid X) e^{\hat{\mu}_{\gamma} \hat{\eta}^{\gamma} / 2}\right)\right|_{\substack{\mu=0 \\
\eta=0}}, \\
\delta_{\alpha \beta}^{L} C(X) & =\left.\frac{1}{2}\left(\frac{\partial^{2}}{\partial \mu^{\alpha} \partial \eta^{\beta}}+\frac{\partial^{2}}{\partial \eta^{\alpha} \partial \mu^{\beta}}\right)\left(C(\hat{\mu} \mid X) e^{\hat{\mu}_{\gamma} \hat{\eta}^{\gamma} / 2}\right)\right|_{\substack{\mu=0 \\
\eta=0}}
\end{aligned}
$$


или

$$
\begin{aligned}
& \delta_{\alpha \beta}^{P} C(X)=\left.\frac{1}{2}\left(W_{1 \alpha}^{\gamma} W_{1 \beta}^{\delta}+\left(W_{2}^{-1}\right)_{\alpha}^{\gamma}\left(W_{2}^{-1}\right)_{\beta}{ }^{\delta}\right) \frac{\partial^{2} C(\mu \mid X)}{\partial \mu^{\gamma} \partial \mu^{\delta}}\right|_{\mu=0}, \\
& \delta_{\alpha \beta}^{L} C(X)=\left.\frac{1}{4}\left(W_{1 \alpha^{\gamma}}{ }^{\gamma} W_{1 \beta}{ }^{\delta}-\left(W_{2}^{-1}\right)_{\alpha}^{\gamma}\left(W_{2}^{-1}\right)_{\beta}{ }^{\delta}\right) \frac{\partial^{2} C(\mu \mid X)}{\partial \mu^{\gamma} \partial \mu^{\delta}}\right|_{\mu=0} .
\end{aligned}
$$

Наконец, используя уравнения движения (6.6) для скалярного поля, имеем

$$
d C(X)=\left.\frac{1}{4} h^{\alpha \beta} \frac{\partial^{2} C(\mu \mid X)}{\partial \mu^{\alpha} \partial \mu^{\beta}}\right|_{\mu=0},
$$

что эквивалентно

$$
2 h^{n}{ }_{, \alpha \beta} \partial_{n} C(X)=\left.\frac{\partial^{2} C(\mu \mid X)}{\partial \mu^{\alpha} \partial \mu^{\beta}}\right|_{\mu=0} .
$$

Используя (4.7) и принимая во внимание (4.11), подстановкой (Б.9) в (Б.7) получаем ответ для действия генераторов AdS-изометрий на скалярное поле в следующем виде:

$$
\begin{aligned}
& \delta_{\alpha \beta}^{P} C(X)=\left(\partial_{m} S_{\alpha \gamma} S_{\beta}^{\gamma}-\partial_{m} S_{\gamma \alpha} S_{\beta}^{\gamma}\right) g^{m n} \partial_{n} C(X), \\
& \delta_{\alpha \beta}^{L} C(X)=\frac{1}{2}\left(\partial_{m} S_{\alpha \gamma} S_{\beta}^{\gamma}+\partial_{m} S_{\gamma \alpha} S_{\beta}^{\gamma}\right) g^{m n} \partial_{n} C(X) .
\end{aligned}
$$

Благодарности. Мы выражаем благодарность С. Карлипу, Р. Р. Мецаеву и И. В. Тютину за полезные комментарии. Работа выполнена при частичной поддержке INTAS (гранты № 03-51-6346, 05-7928), РФФИ (грант № 05-02-17654), Программы поддержки ведущих научных школ (грант № 4401.2006.2).В.Е.Диденко благодарит фонд им. Л. Д. Ландау за финансовую поддержку. В. Е. Диденко и А. С. Матвеев благодарны фонду "Династия" за финансовую поддержку, "Ettore Majorana" Foundation и Center for Scientific Culture, где была выполнена часть работы, за гостеприимство, и Museo Storico della Fisica e Centro Studi e Ricerche Enrico Fermi за награду для начинающих ученых им. Э. Ферми. М. А. Васильев благодарит Ж. Азкарага за гостеприимство во время его посещения Отделения физики Университета г. Валенсия (Испания), где была выполнена часть работы.

\section{Список литературы}

[1] A. Staruszkiewicz, Acta Phys. Polon., 24 (1963), 735.

[2] H. Leutwyler, Nuovo Cimento A, 42 (1966), 159.

[3] S. Deser, R. Jackiw, G. 't Hooft, Ann. Physics, 152 (1984), 220.

[4] S. Deser, R. Jackiw, Ann. Physics, 153 (1984), 405; Commun. Math. Phys., 118 (1988), 495.

[5] G. 't Hooft, Commun. Math. Phys., 117 (1988), 685.

[6] A. Achúcarro, P. K. Townsend, Phys. Lett. B, 180 (1986), 89.

[7] E. Witten, Nucl. Phys. B, 311 (1988), 46; 323 (1989), 113; Commun. Math. Phys., 137 (1991), 29.

[8] S. Carlip, J. Korean Phys. Soc., 28 (1995), S447; gr-qc/9503024.

[9] M. Banados, C. Teitelboim, J. Zanelli, Phys. Rev. Lett., 69 (1992), 1849; hep-th/9204099.

[10] D. Ida, Phys. Rev. Lett., 85 (2000), 3758; gr-qc/0005129. 
[11] M. Banados, M.Henneaux, C. Teitelboim, J. Zanelli, Phys. Rev. D (3), 48 (1993), 1506; gr-qc/9302012.

[12] M. A. Vasiliev, Mod. Phys. Lett. A, 7 (1992), 3689.

[13] S. F. Prokushkin, M. A. Vasiliev, Nucl. Phys. B, 545 (1999), 385; hep-th/9806236.

[14] M. A. Vasiliev, "Higher spin gauge theories: star-product and AdS space", Many Faces of the Superworld, ed. M. Shifman, World Scientific, Singapore, 2000, 533; hep-th/9910096.

[15] E. Sezgin, P. Sundell, Nucl. Phys. B, 762 (2007), 1; hep-th/0508158.

[16] C. Fronsdal, "Massless particles, orthosymplectic symmetry and another type of Kaluza-Klein theory", Essays on Supersymmetry, Math. Phys. Stud., 8, ed. C. Fronsdal, Reidel, Dordrecht, 1986, 163.

[17] I. Bandos, J. Lukierski, D. Sorokin, Phys. Rev. D (3), 61 (2000), 045002; hep-th/9904109.

[18] M. A. Vasiliev, Phys. Rev. D (3), 66 (2002), 066006; hep-th/0106149.

[19] I. Bandos, J. Lukierski, C. Preitschopf, D. Sorokin, Phys. Rev. D (3), 61 (2000), 065009; hep-th/9907113.

[20] V.E. Didenko, M. A. Vasiliev, J. Math. Phys., 45 (2004), 197; hep-th/0301054.

[21] M. Plyushchay, D. Sorokin, M. Tsulaia, JHEP, 04 (2003), 013; hep-th/0301067.

[22] М. А. Васильев, О. В. Шейнкман, ТМФ, 128:3 (2001), 378; hep-th/0103208.

[23] K. Ghoroku, A. L. Larsen, Phys. Lett. B, 328 (1994), 28; hep-th/9403008.

[24] I. Ichinose, Y. Satoh, Nucl. Phys. B, 447 (1995), 340; hep-th/9412144.

[25] S. Das, A. Dasgupta, JHEP, 10 (1999), 025; hep-th/9907116.

[26] D. Birmingham, I. Sachs, S. N. Solodukhin, Phys. Rev. Lett., 88 (2002), 151301; hep-th/0112055.

[27] I. Bars, M. Günaydin, Commun. Math. Phys., 91 (1983), 31.

[28] M. A. Vasiliev, Class. Quantum Grav., 8 (1991), 1387.

[29] И. С. Градштейн, И. М. Рыжик, Таблицы интегралов, сумм, рядов и произведений, Физматгиз, М., 1963.

[30] J. Gamboa, F. Méndez, Class. Quantum Grav., 18 (2001), 225; hep-th/0006020.

[31] S. Lepe, F. Méndez, J. Saavedra, L. Vergara, Class. Quantum Grav., 20 (2003), 2417; hep-th/0302035.

[32] J. Troost, JHEP, 09 (2002), 041; hep-th/0206118.

[33] Н.Я. Виленкин, Специальные функции и теория представлений групп, Наука, М., 1991.

[34] O. Coussaert, M. Henneaux, Phys. Rev. Lett., 72 (1994), 183; hep-th/9310194. 